\title{
Monitoring a PGD solver for parametric power flow problems with goal-oriented error assessment
}

\author{
R. García-Blanco ${ }^{1}$, P. Díez ${ }^{1,0}$, D. Borzacchiello ${ }^{2}$, and F. Chinesta ${ }^{2}$ \\ ${ }^{1}$ Laboratori de Càlcul Numèric (LaCàN), Universidad Politécnica de Cataluña, \\ Barcelona, Spain \\ ${ }^{2}$ Institut de Calcul Intensif (ICI), École Centrale de Nantes, Nantes, France
}

\begin{abstract}
The parametric analysis of electric grids requires carrying out a large number of Power Flow computations. The different parameters describe loading conditions and grid properties. In this framework, the Proper Generalized Decomposition (PGD) provides a numerical solution explicitly accounting for the parametric dependence. Once the PGD solution is available, exploring the multidimensional parametric space is computationally inexpensive. The aim of this paper is to provide tools to monitor the error associated with this significant computational gain and to guarantee the quality of the PGD solution. In this case, the PGD algorithm consists in three nested loops that correspond to 1) iterating algebraic solver, 2) number of terms in the separable greedy expansion and 3) the alternated directions for each term. In the proposed approach, the three loops are controlled by stopping criteria based on residual goal-oriented error estimates. This allows one for using only the computational resources necessary to achieve the accuracy prescribed by the end-user. The paper discusses how to compute the goal-oriented error estimates. This requires linearizing the error equation and the Quantity of Interest to derive an efficient error representation based on an adjoint problem. The efficiency of the proposed approach is demonstrated on benchmark problems.
\end{abstract}

\section{Introduction}

The Power Flow problem models the response of electric grids under quasi-static loading conditions (dynamic effects are neglected). It consists in solving a nonlinear system of algebraic equations providing the complex voltages in each bus (the nodes of the grid are called buses) and for each phase.

The standard solvers for the Power Flow problem are based on the classical methodologies like Newton-Raphson's (NR) [1, 2] and Gauss-Seidel (GS) [3, 4] but also on specific approaches like Fast Decoupled Load Flow (FDLF) [5, 6] or the primitive methods based on admittance or impedance matrices [7, 8, 9, 10, 11, 12], for instance the $Z$ bus method [13]. See [14] for a review. Different strategies have also been devised to avoid the assembly of the Jacobian

\footnotetext{
${ }^{0}$ E-mail: pedro.diez@upc.edu
} 
when the dimension of the problem is considerably large. These include Jacobian-free methods [15], Newton-Krylov methods, [16] or partial Jacobian update variants, [17, 18] among others $[19,20,21,22]$. Other methods address the problem of finding the physical or high voltage solution and avoid nonphysical solutions. For instance, the Holomorphic Embedding Load Flow Method (HELM) [23] or others based on truncated Taylor expansions [24, 25]. Recently, a family of iterative solvers converging to the operative solution was proposed in [26].

Solving the parametric form of the Power Flow problem is of special importance to obtain simulations for operational control and optimization of the electric grids (optimization refers to the design phase but also in modifications like adding new power devices). This requires accounting for a large number of different scenarios that are typically parameterized. The computational burden of such families of parametric problems explodes with the number of parameters (actually, with the number of parametric dimensions). In this context, Reduced Order Models are especially indicated to remedy the curse of dimensionality. In particular the Proper Generalized Decomposition (PGD) providing an explicit expression for the parametric dependence is used in this framework in [27] and [28].

The drastic cost savings associated with PGD may lead to a reduction in accuracy and, consequently, an error assessment strategy is needed to control the quality of the solution and as a stopping criterion for the enrichment process. This issue has already been addressed in different fields $[29,30]$. Particularly, we focus on analyzing the error committed when calculating the system losses solving the Parametric Power Flow Problem using PGD. Thus, the loss functional is taken as the Quantity of Interest to be analyzed.
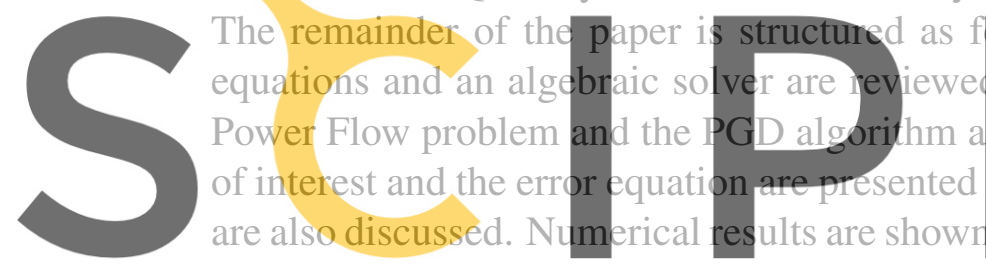

are also discus

Register for free at https//www.scipedia.com to download the version without the watermark 2 The Power Flow problem and an algebraic solver

\subsection{Governing Equations}

The unknowns of the Power Flow problem are the voltages and nodal intensities at the nodes of an electric grid. These unknowns are collected in vectors of $n$ complex components, $V \in \mathbb{C}^{n}$ and $I \in \mathbb{C}^{n}$, where $n$ is the number of degrees of freedom. For a three-phase distribution system, $n$ is three times the number of buses.

The input data characterizing the Power Flow problem is the following:

- The topology of the grid, described by the number of lines, the number of buses and their connectivity.

- The complex powxer source vector $S \in \mathbb{C}^{n}$, describing the power supplied and/or extracted at each phase of each node.

- The admittance matrix $\mathbf{Y} \in \mathbb{C}^{n \times n}$ including the material characteristics of the devices conforming the grid (wires and other system devices like transformers). Note that the sparsity of $\mathrm{Y}$ is associated with the lines-buses connectivity. 
- The vector $I_{0} \in \mathbb{C}^{n}$ accounting for the current originated by the slack node. Introducing a slack node is necessary to garantee the solvability of the problem, see $[31,32,33]$.

At each bus, the nonlinear relation between the voltage, the current and the complex power is provided by the following equation:

$$
S=V \odot I^{*},
$$

where $I^{*}$ denotes the complex conjugate of the current vector $I$, and the symbol $\odot$ denotes the Hadamard product of vectors (component-wise product).

Thus, Kirchhoff's law leads to the following algebraic system of equations:

$$
\mathrm{Y} V=I+I_{0}
$$

which, using (1) results in a nonlinear algebraic system of equations for the unknown $V$ :

$$
\mathbf{Y}=S^{*} \oslash V^{*}+I_{0}
$$

where the symbol $\oslash$ denotes the component-wise quotient between vectors. Note that this equation is also seen as $V^{*} \odot\left(\mathbf{Y} V-I_{o}\right)=S^{*}$.

The remainder of the paper is devoted to discuss how to solve this equation, first in the simple form of (3), then in a parametric version.

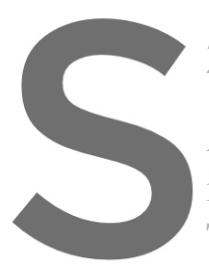

\subsection{An algebraic solve \\ As mentioned in section \\ iterative procedure that \\ The idea comes from ob
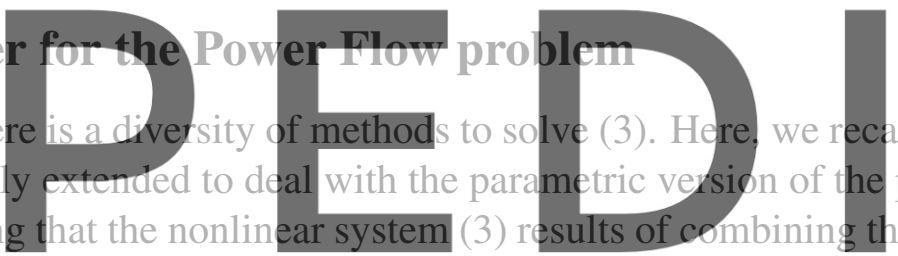

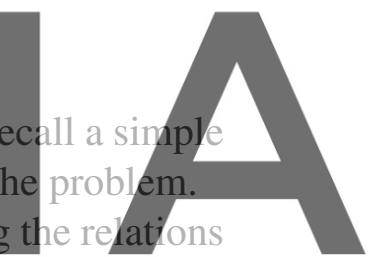

(1) (which is nonlinear but local) and (2) (which is global but linear). Thus, in a divide and

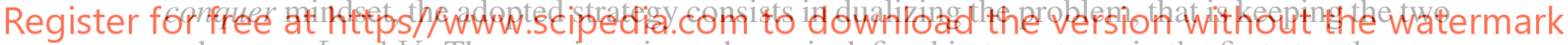

unknowns $I$ and $V$. Then, an iteration scheme is defined in two steps: in the first step the new

approximation for $I$ and $V$ is obtained enforcing (2) and in the second step, using (1). These two steps require defining linear relations between the increments of currents and voltages at each iteration. These relations are given by the search directions $\boldsymbol{\alpha}$ and $\boldsymbol{\beta}$, both considered initially in $\mathbb{C}^{n \times n}$.

Thus, each iteration consists in computing $(V, I)^{[\gamma+1]}$ from the previous approximation $(V, I)^{[\gamma]}$. The first step consists in finding an intermediate approximation $(V, I)^{\left[\gamma+\frac{1}{2}\right]}$ such that

$$
\left\{\begin{array}{l}
I^{\left[\gamma+\frac{1}{2}\right]}-I^{[\gamma]}=\boldsymbol{\alpha}\left(V^{\left[\gamma+\frac{1}{2}\right]}-V^{[\gamma]}\right), \\
\mathbf{Y} V^{\left[\gamma+\frac{1}{2}\right]}=I^{\left[\gamma+\frac{1}{2}\right]}+I_{0} .
\end{array}\right.
$$

Similarly, the second step computes $(V, I)^{[\gamma+1]}$ such that

$$
\left\{\begin{array}{l}
I^{[\gamma+1]}-I^{\left[\gamma+\frac{1}{2}\right]}=\boldsymbol{\beta}\left(V^{[\gamma+1]}-V^{\left[\gamma+\frac{1}{2}\right]}\right), \\
V^{*[\gamma+1]} \odot I^{[\gamma+1]}=S^{*}
\end{array}\right.
$$

This family of algorithms, introduced in [26] is parameterized by the search directions $\boldsymbol{\alpha}$ and $\boldsymbol{\beta}$. A similar approach is used in [34] for nonlinear structural mechanics. The performance of this method is strongly dependent on the choice of the search directions $\boldsymbol{\alpha}$ and $\boldsymbol{\beta}$. For $\boldsymbol{\beta} \rightarrow \infty$ 
and $\boldsymbol{\alpha}=0$, the resulting method is precisely the well known $\mathrm{Z}$ bus method, that can be written as a single step method for $V$, namely

$$
V^{[\gamma+1]}=\mathbf{Y}^{-1}\left(S^{*} \oslash V^{*[\gamma]}+I_{0}\right)
$$

This choice is not the most efficient one for a standard algebraic solver. Nevertheless, in the next sections, the simplicity of this version is preferred for the sake of a simpler presentation in the framework of the parametric version of the problem.

\section{Parametric power flow problem and PGD solver}

\subsection{Parametric problem and separable approximation}

Solving problem (3) as indicated in section 2.2 provides the state of the network for a given configuration of the materials and loads. In practice, analyzing an electric grid requires solving the same problem with a large number of configurations. These configuration are parametrically described. Typical examples of parameters are the location and nominal power (in the following denoted by $q$ and $r$ ) of some Distributed Generator (DG), and the time $t$ that modulates the power $S$. Here, the general form of the Parametric Power Flow problem is described by taking $S$ depending of these three parameters in (3), that is $S(q, r, t)$.

Consequently, the solution of (3) is also depending of these parameters, namely $V(q, r, t)$. In practice, this brings the problem from a simple nonlinear algebraic equation in $\mathbb{C}^{\prime}$ into a multi-
dimensional setup: formally, $V$ is now in $\left[L_{2}\left(I_{q}\right) \times L_{2}\left(I_{r}\right) \times I_{2}\left(I_{t}\right)\right]^{n}$, that is, each component
of $V$ is a function taking values for $(q, r, t) \in I_{q} \times I_{r} \times I_{t}$ where $I_{q}, I_{r}$ and $I_{t}$ are intervals in
$\mathbb{R}$. This multidimensional problem is potentially subject to the curse of dimensiondity, that is
to a dramatic increment of the computational cost with the number of dimensions. This phenomenon is remedied using Reduced Order Models (ROM), see [35, 36, 37, 38, 39, 40] for

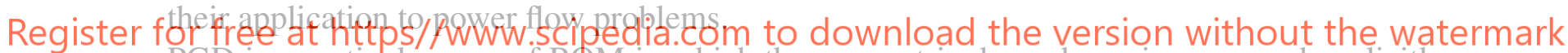
PGD is a particular case of ROM in which the parametric dependence is expressed explicitly.

The PGD approximation $V_{a}$ has a separated form. That means that it is a sum of $M$ terms, each of them being the product of functions only depending on one of the parameters, namely

$$
V(q, r, t) \approx V_{a}(q, r, t)=\sum_{m=1}^{M} \alpha_{V}^{m} V^{m} \mathcal{Q}^{m}(q) \mathcal{R}^{m}(r) \mathcal{T}^{m}(t),
$$

where, for $m=1, \ldots, M, \alpha_{V}^{m}$ are positive scalars, $V^{m} \in \mathbb{C}^{n}$ are the unit vector modes of voltages, and $\mathcal{Q}^{m}(q), \mathcal{R}^{m}(r)$ and $\mathcal{T}^{m}(t)$ are the unit parametric modes. The modes are normalized (to have unit norm) and the positive scalar $\alpha_{V}^{m}$ collects the amplitude of each term.

In practice, the parametric dimensions are discretized in a Finite Element fashion. Let $n_{q}, n_{r}$ and $n_{t}$ denote the number of degrees of freedom discretizing the three parametric dimensions. Thus, function $\mathcal{Q}^{m}(q)$ is identified with vector $\mathcal{Q}^{m} \in \mathbb{C}^{n_{q}}$, similarly vectors $\mathcal{R}^{m} \in \mathbb{C}^{n_{r}}$ and $\mathcal{T}^{m} \in \mathbb{C}^{n_{t}}$ represent functions $\mathcal{R}^{m}(r)$ and $\mathcal{T}^{m}(t)$. Thus, the multivariate function $V_{a}(q, r, t)$ (from $I_{q} \times I_{r} \times I_{t}$ to $\mathbb{C}^{n_{r}}$ ) is also described by a $n \times n_{q} \times n_{r} \times n_{t}$ complex tensor $\mathbf{V}_{a}$, such that

$$
\mathbf{V}_{a}=\sum_{m=1}^{M} \alpha_{V}^{m} V^{m} \otimes \mathcal{Q}^{m} \otimes \mathcal{R}^{m} \otimes \mathcal{T}^{m}
$$


Remark 1. Using finite element approximations of the parametric modes is only a way of numerically describing the 1D functions. For instance, $\mathcal{Q}^{m}$ can be seen just as a set of values of $\mathcal{Q}^{m}(q)$ in some sampling points. The fact of identifying this set of points with a finite element $1 \mathrm{D}$ grid is not strictly necessary in this context but it can be helpful in the case some integral of the nodal modes needs to be computed. For instance, in section 4.2, a quantity of interest based on the integral along a parametric dimension of a function is introduced. Thus, the underlying finite element space, typically $\mathcal{C}^{0}$ linear elements which is the simplest option, allows performing the integrals systematically. Note also that the continuity of the parametric modes is implicitly assumed and therefore the functional spaces where the modes are sought are, in practice, smaller than $L_{2}$.

In order to use PGD, parametric dependence on the input data has also to be expressed in a separable form. Thus, in the following it is assumed that

$$
S(q, r, t)=\sum_{h=1}^{H} \alpha_{S}^{h} S^{m} \breve{Q}^{h}(q) \breve{\mathcal{R}}^{h}(r) \breve{T}^{h}(t),
$$

where $H$ is the number of terms in the $S$ expansion, and for $h=1, \ldots, H, \alpha_{S}^{h}$ are positive scalars, $S^{h} \in \mathbb{C}^{n}$ are the unit vector modes of powers, and $\breve{\mathcal{Q}}^{h}(q), \breve{\mathcal{R}}^{h}(r)$ and $\breve{\mathcal{T}}^{h}(t)$ are the unit parametric modes. Similarly to $(8), S(q, r, t)$ is identified with its tensorial version $\mathbf{S} \in$ $\mathbb{C}^{n \times n_{q} \times n_{r} \times n_{t}}$ that reads
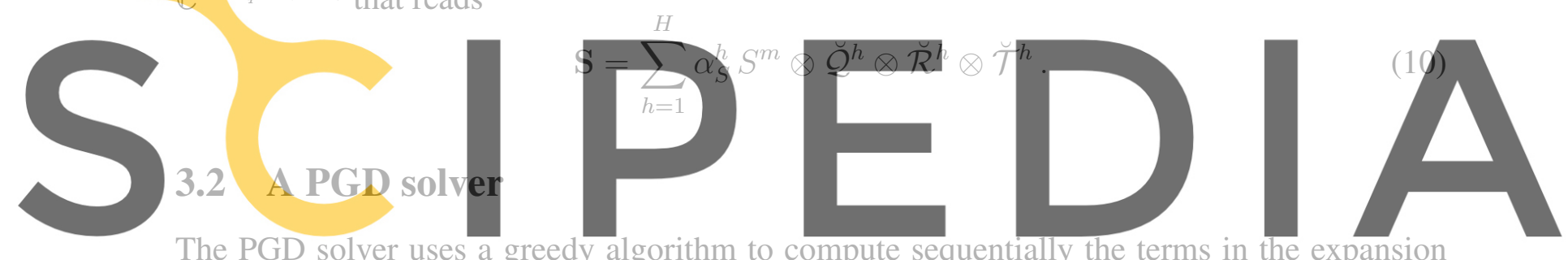

The PGD solver uses a greedy algorithm to compute sequentially the terms in the expansion Register for free its tensorial form (8)) see [41, 42,43 , 44]. Adapting the iterative strategy presented in section 2.2 (the so-called $Z$ bus method) to the parametric context can be summarized in rewriting (6) with the explicit parametric dependence, i.e.

$$
V_{a}^{[\gamma+1]}(q, r, t)=\mathbf{Y}^{-1}\left(S^{*}(q, r, t) \oslash V_{a}^{*[\gamma]}(q, r, t)+I_{0}\right)
$$

For algorithmic purposes, and following the ideas presented in section 2.2, this operation is split into two steps. First, an intermediate quantity $I$ is computed such that

$$
I(q, r, t)=S^{*}(q, r, t) \oslash V_{a}^{*[\gamma]}(q, r, t),
$$

Thus, the second step consists in solving the global (but linear) system, that is in computing

$$
V_{a}^{[\gamma+1]}(q, r, t)=\mathbf{Y}^{-1}\left(I(q, r, t)+I_{0}\right)
$$

Note that in (11), the operations are not as trivial as in their algebraic version. For instance, for $S$ and $V_{a}$ in $\mathbb{C}^{n}$, computing $I=S^{*} \oslash V_{a}$ (which is the operation corresponding to (5) in the adopted choice for $\boldsymbol{\alpha}$ and $\boldsymbol{\beta})$ is a simple division for each component: $[I]_{\ell}=[S]_{\ell}^{*} /[V]_{\ell}^{*}$ for $\ell=1, \ldots, n$. For $V_{a}(q, r, t)$ and $S(q, r, t)$ represented in the separable forms (7) and (9), the operation (12) requires solving a PGD problem. That is (for each iteration $\gamma$ ) to solve a problem of the type: find $I(q, r, t)$ such that $I(q, r, t) \odot V_{a}^{*[\gamma]}(q, r, t)=S^{*}(q, r, t)$. The standard PGD procedure consists in computing sequentially the terms of the PGD expansion of $I(q, r, t)$ 
(loop on $M$ ) and for each term iterate in the alternated directions scheme (this is going to be denoted as a loop on $k$ ).

Thus, in this context, the PGD algorithm involves three nested loops:

- the external one corresponds to the nonlinear solver and iterates in $\gamma$

- the second is the greedy part of the PGD algorithm to solve (12) (loop on the number of terms of the PGD expansion $M$ )

- the inner loop iterates (for $k=1,2, \ldots$ ) in the alternated direction scheme for each of the parametric dimensions.

The global idea of the PGD procedure is described and illustrated in Algorithm 1, see [27] and [28]. The initialization is typically provided after the slack node intensity, $I_{0}$, namely

$$
V_{a}^{[0]}=\mathbf{Y}^{-1} I_{0}
$$

The different stopping criteria based on the user prescribed tolerances are discussed in the next section.

\section{Algorithm 1:}

\section{Data: $I_{0}, S$}

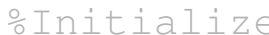
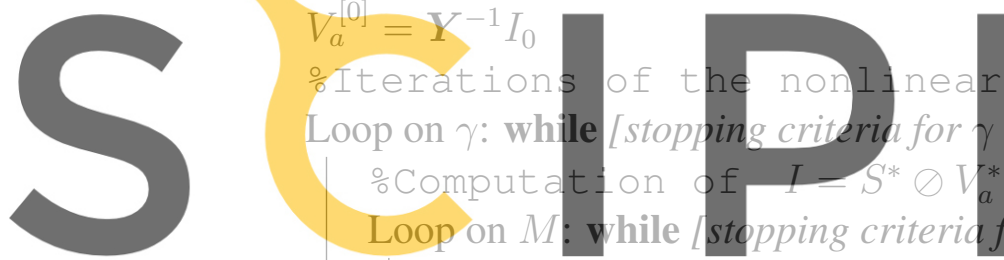

Loop on $M$ :

hile /stopping criteria for $M$ do
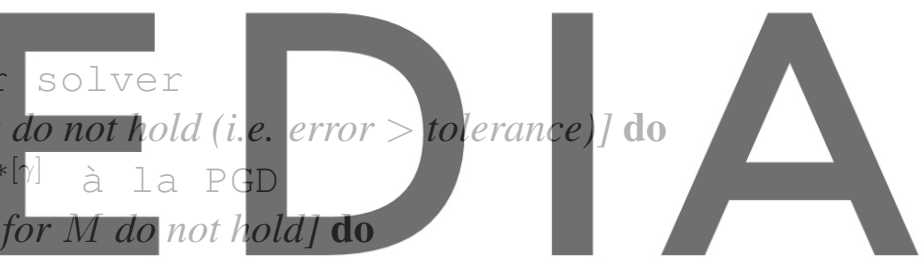

Register for free at https//Awww.scipedia.com to downtoad the version without the watermark

oIterations for alternated directions

Loop on $k$ : while [stopping criteria for $k$ do not hold] do

Compute $\left(V^{M}\right)^{k+1}$ from $\left(\mathcal{Q}^{M}(q)\right)^{k},\left(\mathcal{R}^{M}(r)\right)^{k}$ and $\left(\mathcal{T}^{M}(t)\right)^{k}$;

Compute $\left(\mathcal{Q}^{M}(q)\right)^{k+1}$ from $\left(V^{M}\right)^{k+1},\left(\mathcal{R}^{M}(r)\right)^{k}$ and $\left(\mathcal{T}^{M}(t)\right)^{k}$;

Compute $\left(\mathcal{R}^{M}(r)\right)^{k+1}$ from $\left(V^{M}\right)^{k+1},\left(\mathcal{Q}^{M}(q)\right)^{k+1}$ and $\left(\mathcal{T}^{M}(t)\right)^{k}$;

Compute $\left(\mathcal{T}^{M}(t)\right)^{k+1}$ from $\left(V^{M}\right)^{k+1},\left(\mathcal{Q}^{M}(q)\right)^{k+1}$ and $\left(\mathcal{R}^{M}(r)\right)^{k+1}$;

을 $V_{a}^{[\gamma+1]}=\boldsymbol{Y}^{-1}\left(I+I_{0}\right)$

$V^{m} \leftarrow \boldsymbol{Y}^{-1} V^{m}$ for $m=1, \ldots, M$;

$V_{a}^{[\gamma+1]}=\mathbf{Y}^{-1} I+V_{a}^{[0]}$

\section{Error assessment}

This section aims at introducing the concept of error assessment in the context of the power flow and the Parametric Power Flow problem. Besides the standard stopping criteria for the solver described above, novel criteria are devised using goal-oriented error assessment. 


\subsection{Algebraic formulation of error assessment}

\subsubsection{Error equation.}

Vector $V$ is the actual solution of the problem and $V_{a}$ is an approximation, both in $\mathbb{C}^{n}$. The error is readily defined as

$$
E=V-V_{a} .
$$

The residual of equation (3) associated with $V_{a}$ (also in $\mathbb{C}^{n}$ ) reads

$$
R\left(V_{a}\right)=S^{*}-V_{a}^{*} \odot\left(\mathbf{Y} V_{a}-I_{0}\right) .
$$

The error equation is derived from the identity $R(V)=0$, that is $R\left(V_{a}+E\right)=0$. The idea is to linearize $R(\cdot)$ noting that $R\left(V_{a}\right)$ is computable once $V_{a}$ is obtained. Expanding the expression of $R(\cdot)$, it is found that

$$
\begin{array}{r}
R(V)=R\left(V_{a}+E\right)=S^{*}-\left(V_{a}^{*}+E^{*}\right) \odot\left(\mathbf{Y}\left(V_{a}+E\right)-I_{0}\right)= \\
=S^{*}-V_{a}^{*} \odot\left(\mathbf{Y} V_{a}-I_{0}\right)-V_{a}^{*} \odot \mathbf{Y} E-E^{*} \odot\left(\mathbf{Y} V_{a}-I_{0}\right)-E^{*} \odot \mathbf{Y} E .
\end{array}
$$

In order to obtain a linear equation for the error, the quadratic term $E^{*} \odot \mathrm{Y} E$ is neglected, that is

$$
R(V)=R\left(V_{a}\right)-V_{a}^{*} \odot \mathrm{Y} E-E^{*} \odot\left(\mathbf{Y} V_{a}-I_{0}\right)=R\left(V_{a}\right)-\mathrm{A} E-\mathrm{B}^{*}
$$

where $\mathbf{A}=\operatorname{Diag}\left(V_{a}^{*}\right) \mathbf{Y}$ and $\mathbf{B}=\operatorname{Diag}\left(\mathbf{Y} V_{a}-I_{0}\right)$ are matrices in $\mathbb{C}^{n \times n}$. The operator $\operatorname{Diag}(\cdot)$

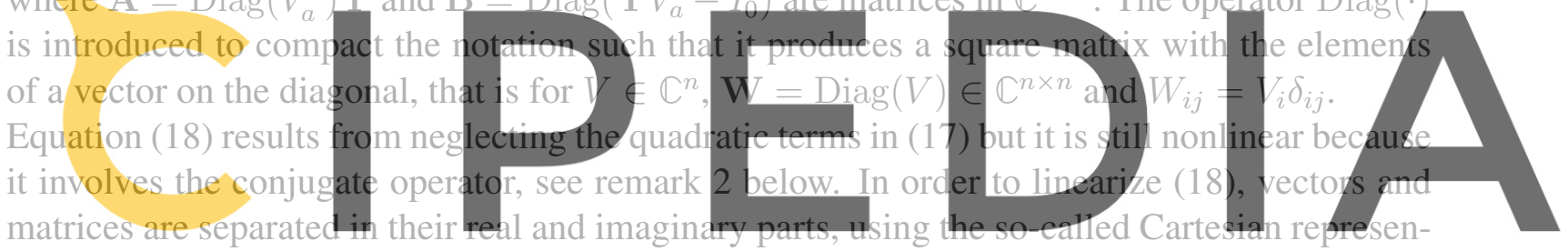

tation. Thus, equation (18) is rewritten as a linear system of $2 n$ real equations and unknowns,

where the matrix $\mathbf{C} \in \mathbb{R}^{2 n \times 2 n}$, and vectors $\hat{R}\left(V_{a}\right)$ and $\hat{E}$ in $\mathbb{R}^{2 n}$ are the real valued representations (duplicating dimensions) of the complex matrices and vectors in (18), that is

$$
\mathbf{C}=\left(\begin{array}{cc}
\Re(\mathbf{A})+\Re(\mathbf{B}) & -\mathfrak{I}(\mathbf{A})+\Im(\mathbf{B}) \\
\mathfrak{I}(\mathbf{A})+\mathfrak{I}(\mathbf{B}) & \Re(\mathbf{A})-\Re(\mathbf{B})
\end{array}\right), \hat{R}\left(V_{a}\right)=\left(\begin{array}{c}
\Re\left(R\left(V_{a}\right)\right) \\
\mathfrak{I}\left(R\left(V_{a}\right)\right)
\end{array}\right) \text { and } \hat{E}=\left(\begin{array}{c}
\Re(E) \\
\mathfrak{I}(E)
\end{array}\right)
$$

where $\Re(\cdot)$ and $\mathfrak{I}(\cdot)$ stand for the real and the imaginary part of the matrix or vector.

Remark 2. The nonlinearity in equation (18) arises from the fact that it involves the conjugate of the error. The conjugate operator is nonlinear and also non-holomorphic (the CauchyRiemann equations are obviously not fulfilled in this case). The non-holomorphic character of the resulting expression precludes using the linearization via the Laurent series truncation strategy (similar to truncating the Taylor expansion for the real-valued functions). The separation in real and imaginary part presented above is an effective alternative to linearize the resulting equations. 


\subsubsection{Error representation via adjoint problem and error estimates.}

It is assumed that the Quantity of Interest (QoI) in the simulation are the energy or power losses. This is a standard choice for grid optimization, where energy losses are the objective quantity to be minimized. In general, given a generic vector of voltages $W$, the positive number representing the losses associated with this vector is:

$$
l(W)=\Re\left(W^{* \top} \mathbf{Y}_{\mathcal{L}} W\right)
$$

where the matrix $\mathbf{Y}_{\mathcal{L}}$ coincides with the admittance matrix $\mathbf{Y}$ almost everywhere. The difference between $\mathrm{Y}_{\mathcal{L}}$ and $\mathrm{Y}$ corresponds to the admittance values of the generators which in $\mathrm{Y}_{\mathcal{L}}$ are taken to be 0 . In other words, $\mathbf{Y}_{\mathcal{L}}$ is the admittance matrix corresponding to the grid, accounting for all the lines and buses but not including the terms associated with the generators. Note that the form $l(\cdot)$ is nonlinear and has to be linearized in order to define a goal-oriented error assessment strategy.

The expression of the losses in terms of the approximated solution and the error reads

$$
\begin{aligned}
l(V) & =\Re\left(V^{* \top} \mathbf{Y}_{\mathcal{L}} V\right)=\Re\left(\left(V_{a}^{*}+E^{*}\right)^{\top} \mathbf{Y}_{\mathcal{L}}\left(V_{a}+E\right)\right) \\
& =\Re\left(V_{a}^{* \top} \mathbf{Y}_{\mathcal{L}} V_{a}\right)+\Re\left(V_{a}^{* \top} \mathbf{Y}_{\mathcal{L}} E\right)+\Re\left(E^{* \top} \mathbf{Y}_{\mathcal{L}} V_{a}\right)+\Re\left(E^{* \top} \mathbf{Y}_{\mathcal{L}} E\right) .
\end{aligned}
$$

Following the same procedure as in section 4.1.1, the quadratic term is neglected:
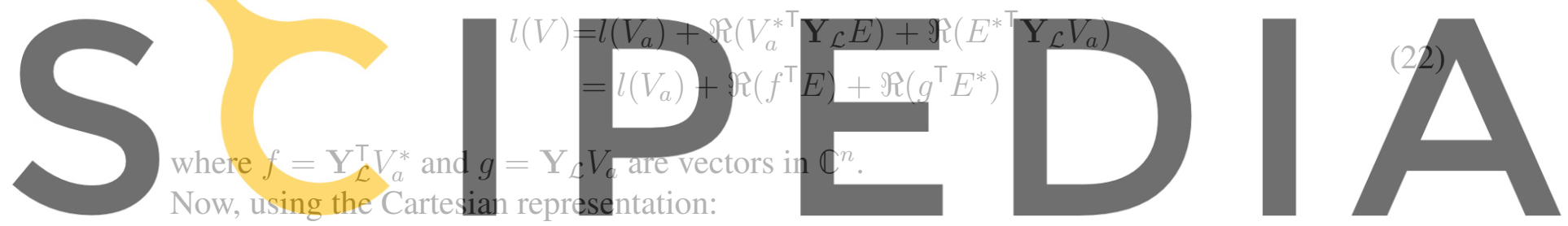

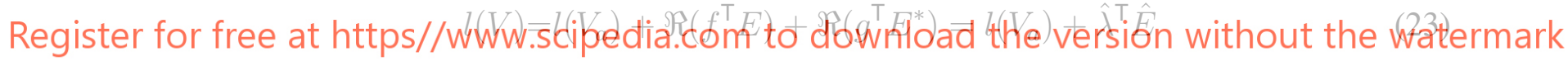

where

$$
\hat{\lambda}=\left(\begin{array}{c}
\Re(f)+\Re(g) \\
-\mathfrak{I}(f)+\mathfrak{I}(g)
\end{array}\right) \in \mathbb{R}^{2 n}
$$

That is, the linear approximation for the error in the Quantity of Interest reads

$$
E_{Q o I}=l(V)-l\left(V_{a}\right)=\hat{\lambda}^{\top} \hat{E}
$$

The following auxiliary problem (referred to as dual or adjoint problem) is introduced in order to obtain a representation of the error in the quantity of interest:

$$
\mathbf{C}^{\top} \hat{\rho}=\hat{\lambda}
$$

The solution of this problem, $\hat{\rho}$, is a real vector of dimension $2 n$. This is a classical strategy that has been applied to different problems in the field of error estimation for reduced order models, see $[29,45,46]$. Assuming that the linearization of equation (21) holds, using $\hat{\rho}$ and (19), the error in the QoI is readily represented as:

$$
E_{Q o I}=\hat{\lambda}^{\top} \hat{E}=\hat{\rho}^{\top} \mathbf{C} \hat{E}=\hat{\rho}^{\top} \hat{R}\left(V_{a}\right)
$$


The matrix $\mathbf{C}$ and the vectors $\hat{R}\left(V_{a}\right), \hat{\lambda}$ and $\hat{\rho}$ are computable but all of them depend on the approximation $V_{a}$ at every iteration. This means that the computational cost for computing the adjoint problem at one iteration is almost the same than the cost of a PGD solver iteration.

Nevertheless, this can be simplified because $\mathbf{C}$ and the vector $\hat{\lambda}$ become stable after a few iterations: in practice, they are constant along the iterative process, and therefore the corresponding dual solution does not change along the iterative process. That is, $\hat{\rho}$ does not change significantly with the $\gamma$ iterations and it is not necessary to solve the dual problem at every iteration. This is related to the fact that, once the approximation enters the asymptotic range, one should expect that $V_{a}$ suffers slight perturbations along the $\gamma$ iterations. Note also that $\mathbf{C}$ and $\hat{\lambda}$ depend linearly on $V_{a}$ and therefore the perturbations in the left and right side of (25) do cancel each other producing almost the same solution $\hat{\rho}$. This property is also observed in the numerical experiments, see Figures $2 \mathrm{a}$ and $2 \mathrm{~b}$ in section 5.1. Once vector $\hat{\rho}$ is obtained, computing the error in the quantity of interest with (26) requires only computing the residual $\hat{R}\left(V_{a}\right)$ which is affordable in terms of computational time.

\subsection{Error assessment in the parametric problem}

This section aims at describing the methodology to assess the error in the framework of the parametric problem described in section 3. For the sake of a simpler presentation, we omit here the dependence on $q$ and $t$ (it is equivalent to take $n_{q}, n_{t}=1$ ). However, this is not a loss of generality because the behaviour of the $q$ and $t$ parametric dimensions is analogous to the $r$

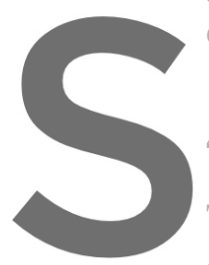
dimension.

4.2.1 Error equation, Taking the tensorial representation is here omitted), $V_{a} \in C^{n \times n_{r}}$ is such that
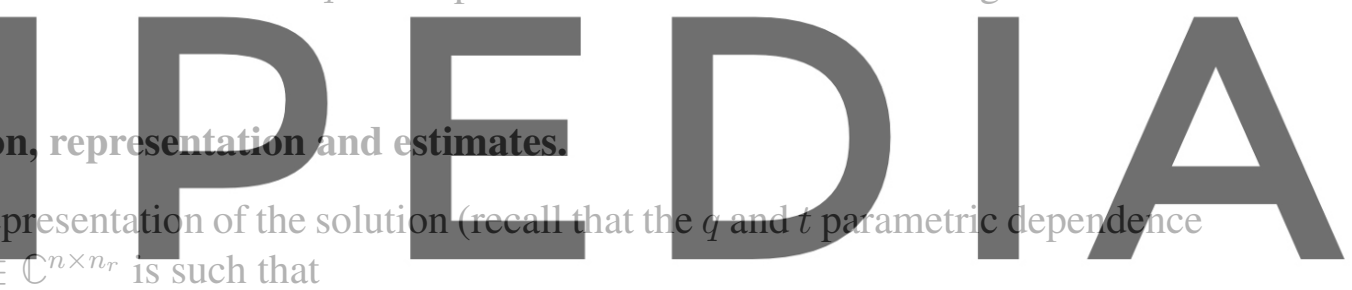

Register for free at https//www.scipedia.con to download the version without the watermark

$$
\mathrm{V}_{a}=\sum_{m} \alpha_{V}^{m} V^{m} \otimes \mathcal{R}^{m}
$$

the error and the residual are also complex matrices in $\mathbb{C}^{n \times n_{r}}$,

$$
\begin{gathered}
\mathbf{E}=\mathbf{V}-\mathbf{V}_{a}, \\
\mathbf{R}(\mathbf{V})=\mathbf{S}^{*}-\mathbf{V}^{*} \odot\left(\boldsymbol{Y} \mathbf{V}-\mathbf{I}_{0}\right) .
\end{gathered}
$$

In the parametric setup, the QoI is taken as the integration with respect to the parametric dimensions (here, only $r$ ) of some nonparametric QoI $l(\cdot)$, namely

$$
L\left(V_{a}(r)\right)=\int_{r} l\left(V_{a}(r)\right) d r .
$$

Note that if normalized by the measure of $I_{r}, L\left(V_{a}(r)\right)$ can be seen as the average value of $l\left(V_{a}(r)\right)$.

Recalling the identity between the functional and tensorial representations in (7) and (8), the integral along $r$ is also determined by the mass matrix $\mathbf{M}_{r}$ (associated with the 1D mesh discretizing $I_{r}$ ) multiplied by vector $\mathbb{1}_{n_{r}}=[1,1, \ldots, 1]^{\top} \in \mathbb{C}^{n_{r}}$ :

$$
L\left(\mathbf{V}_{a}\right)=\mathbb{1}_{n_{r}}^{\top} \mathbf{M}_{r} l\left(\mathbf{V}_{a}\right),
$$


where $l(\cdot)$ is now the generalization to the tensor representation of the operator introduced in equation (20), producing a vector of $n_{r}$ components, namely

$$
l\left(\mathbf{V}_{a}\right)=\operatorname{diag}\left(\Re\left(\mathbf{V}_{a}^{* \top} \mathbf{Y}_{\mathcal{L}} \mathbf{V}_{a}\right)\right)
$$

where the operator $\operatorname{diag}(\cdot)$ maps the elements of the diagonal of the input matrix of size $n_{r} \times n_{r}$ into a column vector of size $n_{r}$. Similarly as in (22),

$$
\begin{aligned}
l(\mathbf{V}) & =l\left(\mathbf{V}_{a}\right)+\Re\left(\mathbf{V}_{a}^{* \mathrm{~T}} \mathbf{Y}_{\mathcal{L}} \mathbf{E}\right)+\operatorname{diag}\left(\Re\left(\mathbf{E}^{* \mathrm{~T}} \mathbf{Y}_{\mathcal{L}} V_{a}\right)\right)+\operatorname{diag}\left(\Re\left(\mathbf{E}^{* \mathrm{~T}} \mathbf{Y}_{\mathcal{L}} \mathbf{E}\right)\right) \\
& \approx l\left(\mathbf{V}_{a}\right)+\operatorname{diag}(\Re(\mathbf{F E}))+\operatorname{diag}\left(\Re\left(\mathbf{G E}^{*}\right)\right)
\end{aligned}
$$

where $\mathbf{F}=\mathbf{V}_{a}^{* \top} \mathbf{Y}_{\mathcal{L}}$ and $\mathbf{G}=\left(\mathbf{Y}_{\mathcal{L}} \mathbf{V}_{a}\right)^{\top}$ are matrices in $\mathbb{C}^{n_{r} \times n}$. Assuming that the approximation holds and using the Cartesian representation, this equation is rewritten as:

$$
l(\mathbf{V})=l\left(\mathbf{V}_{a}\right)+\operatorname{diag}\left(\hat{\boldsymbol{\lambda}}_{p}^{\top} \hat{\mathbf{E}}\right)
$$

where

$$
\hat{\boldsymbol{\lambda}}_{p}=\left(\begin{array}{c}
\Re(\mathbf{F})+\Re(\mathbf{G}) \\
-\Im(\mathbf{F})+\Im(\mathbf{G})
\end{array}\right), \hat{\mathbf{E}}=\left(\begin{array}{c}
\Re(\mathbf{E}) \\
\Im(\mathbf{E})
\end{array}\right) \in \mathbb{R}^{2 n \times n_{r}}
$$

Using the tensor contraction notation, equation (34) becomes:
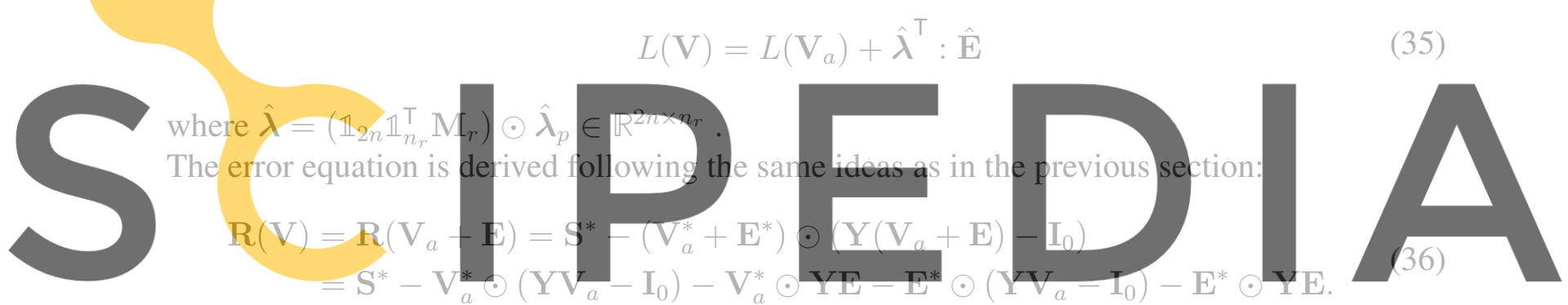

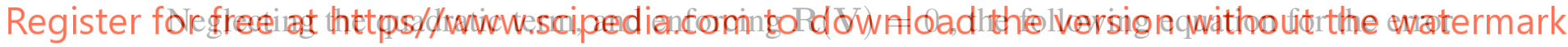
follows

$$
\mathbf{V}_{a}^{*} \odot \mathbf{Y E}+\mathbb{E}^{*} \odot\left(\mathbf{Y V}_{a}-\mathbf{I}_{0}\right)=\mathbf{R}\left(\mathbf{V}_{a}\right)
$$

Taking every column of the matrix $\mathbf{V}_{a}$, it is possible to build two tensors $\underline{\mathbf{A}}(\cdot, \cdot, \ell)=\operatorname{Diag}\left(\mathbf{V}_{a}^{*}(\cdot, \ell)\right) \mathbf{Y}$ and $\underline{\mathbf{B}}(\cdot, \cdot, \ell)=\operatorname{Diag}\left(\left(\mathbf{Y V}_{a}(\cdot, \ell)-\mathbf{I}_{0}(\cdot, \ell)\right)\right) \mathbf{Y}$ for $\ell=1, \ldots, n_{r}$ in $\mathbb{C}^{n \times n \times n_{r}}$. Thus, (37) is rewritten as:

$$
\underline{\mathbf{A}} \odot \mathbf{E}+\underline{\mathbf{B}} \odot \mathbf{E}^{*}=\mathbf{R}\left(\mathbf{V}_{a}\right)
$$

where the operation $\odot$ denotes a contraction of one index and a Hadamard product in another index. For instance, in the particular case of $\underline{\mathbf{A}} \in \mathbb{C}^{n \times n \times n_{r}}$ and $\mathbf{E} \in \mathbb{C}^{n \times n_{r}}$, it reads

$$
[\underline{\mathbf{A}} \odot \mathbf{E}]_{i \ell}=\sum_{j=1}^{n} \underline{\mathbf{A}}_{i j \ell} \mathbf{E}_{j \ell}, \text { with no sum on } \ell \text {. }
$$

Note that the definition is general for the field $(\mathbb{C}$ can be replaced by $\mathbb{R})$ and for the dimensions of the tensors, the only restriction being that the two last indices of tensor $\underline{\mathbf{A}}$ have the same range as the the two indices of tensor $\mathbf{E}$.

Using the Cartesian representation, the equation becomes linear:

$$
\underline{\mathbf{C}} \odot \hat{\mathbf{E}}=\hat{\mathbf{R}}\left(\mathbf{V}_{a}\right)
$$


where tensor $\underline{\mathbf{C}} \in \mathbb{R}^{2 n \times 2 n \times n_{r}}$ is:

$$
\underline{\mathbf{C}}(\cdot, \cdot, \ell)=\left(\begin{array}{cc}
\Re(\underline{\mathbf{A}}(\cdot, \cdot, \ell))+\Re(\underline{\mathbf{B}}(\cdot, \cdot, \ell)) & -\mathfrak{I}(\underline{\mathbf{A}}(\cdot, \cdot, \ell))+\mathfrak{I}(\underline{\mathbf{B}}(\cdot, \cdot, \ell)) \\
\mathfrak{I}(\underline{\mathbf{A}}(\cdot, \cdot, \ell))+\mathfrak{I}(\underline{\mathbf{B}}(\cdot, \cdot, \ell)) & \Re(\underline{\mathbf{A}}(\cdot, \cdot, \ell))-\Re(\underline{\mathbf{B}}(\cdot, \cdot, \ell))
\end{array}\right), \forall \ell=1, \ldots, n_{r} .
$$

and

$$
\hat{\mathbf{R}}=\left(\begin{array}{c}
\Re(\mathbf{R}) \\
\mathfrak{I}(\mathbf{R})
\end{array}\right) \in \mathbb{R}^{2 n \times n_{r}}
$$

The dual problem is readily introduced as:

$$
\underline{\mathbf{C}}^{\top} \odot \hat{\boldsymbol{\rho}}=\hat{\boldsymbol{\lambda}}
$$

where $\underline{\mathbf{C}}^{\top}(\cdot, \cdot, \ell)=\underline{\mathbf{C}}(\cdot, \cdot, \ell)^{\top}, \forall \ell$ (transposing only the two first dimensions of the tensor). Hence the error in the quantity of interest using equation (40) is:

$$
\begin{aligned}
E_{Q o I} & =L(\mathbf{V})-L\left(\mathbf{V}_{a}\right)=\hat{\boldsymbol{\lambda}}^{\top}: \hat{\mathbf{E}}=\hat{\boldsymbol{\lambda}}^{\top}:\left(\underline{\mathbf{C}}^{\dagger} \odot \hat{\mathbf{R}}\left(\mathbf{V}_{a}\right)\right)= \\
& =\hat{\boldsymbol{\rho}}^{\top}: \hat{\mathbf{R}}\left(\mathbf{V}_{a}\right)
\end{aligned}
$$

where $\underline{\mathbf{C}}^{\dagger}(\cdot, \cdot, \ell)=\underline{\mathbf{C}}^{-1}(\cdot, \cdot, \ell), \forall \ell$ (sectionally inverting the two first dimensions of the tensor). The previous identity is straightforwardly derived by analyzing the component-wise expression:

$$
E_{Q o I}=\sum_{i, \ell} \hat{\boldsymbol{\lambda}}_{\ell i} \hat{\mathbf{E}}_{i \ell}=\sum_{i, j, \ell} \hat{\boldsymbol{\lambda}}_{\ell i} \underline{\mathbf{C}}_{i j \ell}^{\dagger} \hat{\mathbf{R}}\left(\mathbf{V}_{a}\right)_{j \ell}=\sum_{j, \ell} \hat{\boldsymbol{\rho}}_{j \ell} \hat{\mathbf{R}}\left(\mathbf{V}_{a}\right)_{j \ell}
$$

using the definition of the dual problem in equation (41).

The error representation provided in (42) describes the scalar $E_{Q o I}$ as the double contraction of $\hat{\boldsymbol{\rho}}^{\top}$ and $\hat{\mathbf{R}}\left(\mathbf{V}_{a}\right)$, both $n \times n_{r}$ tensors. This is because the residual error equation (40) is in fact a set of $n_{r}$ algebraic residual equations similar to (19), one for each possible value of parameter $r$. The same occurs with the adjoint problem (41), which can be seen as a collection of $n_{r}$ algebraic adjoint problems like (25).

The error assessment technique using the solution $\hat{\boldsymbol{\rho}}^{\top}$ of (41) and the error representation (42) is in practice computationally unaffordable. This is due to the multidimensional character of both $\hat{\boldsymbol{\rho}}^{\top}$ and $\hat{\mathbf{R}}\left(\mathbf{V}_{a}\right)$, which are tensors of order $n \times n_{q} \times n_{r} \times n_{t}$. Moreover, once $\hat{\boldsymbol{\rho}}^{\top}$ and $\hat{\mathbf{R}}\left(\mathbf{V}_{a}\right)$ are obtained, all the tensorial dimensions must be contracted (this requires four nested loops) to compute the scalar quantity $E_{Q o I}$. In the following, we introduce a numerical strategy that condensates all the parametric dimensions in order to devise an amenable error assessment methodology.

Thus, the QoI introduced in (30) (or its matrix form (31)) is integrating the effect of the parametric dimensions and one expects the resulting problem to depend only on the physical dimension (represented here by the vector of voltages of size $n$ ). Accordingly, we expect providing an error representation having the form

$$
E_{Q o I}=\left(\hat{\rho}^{A}\right)^{\top} \hat{R}^{A}\left(\mathbf{V}_{a}\right)
$$

where $\hat{\rho}^{A}$ and $\hat{R}^{A}\left(\mathbf{V}_{a}\right)$ are vectors in $\mathbb{R}^{2 n}$ that have to be obtained condensing the parametric dimensions (here, integrating with respect to parameter $r$ ). 
The condensation of $\hat{\mathbf{R}}\left(\mathbf{V}_{a}\right) \in \mathbb{R}^{2 n \times n_{r}}$ and $\underline{\mathbf{C}} \in \mathbb{R}^{2 n \times 2 n \times n_{r}}$ into $\hat{R}^{A}\left(\mathbf{V}_{a}\right) \in \mathbb{R}^{2 n}$ and $\mathbf{C}^{A} \in$ $\mathbb{R}^{2 n \times 2 n}$ (superscript $A$ is used to denote that the quantities are condensed into an accumulated value) is readily obtained by just integrating the parametric dimension, namely

$$
\hat{R}^{A}\left(\mathbf{V}_{a}\right)=\int_{r} \hat{R}(\cdot, r) d r=\hat{\mathbf{R}}\left(\mathbf{V}_{a}\right) \mathbf{M}_{r} \mathbb{1}_{n_{r}}
$$

and

$$
\mathbf{C}^{A}=\int_{r} \underline{\mathbf{C}}(\cdot, \cdot, r) d r=\underline{\mathbf{C}} \mathbf{M}_{r} \mathbb{1}_{n_{r}} .
$$

It is assumed that there exists some vector $\hat{E}^{A} \in \mathbb{R}^{2 n}$, representing an average value of $\hat{\mathbf{E}}(\cdot, r)$, such that

$$
\int_{r} \underline{\mathbf{C}}(\cdot, \cdot, r) \odot \hat{\mathbf{E}}(\cdot, r) d r=\mathbf{C}^{A} \hat{E}^{A}
$$

Consequently, the equation for the mean error $\hat{E}^{A}$ is precisely the following linear system of dimension $2 n$

$$
\mathbf{C}^{A} \hat{E}^{A}=\hat{R}^{A}\left(\mathbf{V}_{a}\right) .
$$

Note that the existence of vector $\hat{E}^{A}$ is guaranteed by the integral Mean Value Theorem applied to the left-hand-side of (40), under the hypothesis of having a continuous dependence of $\hat{\mathbf{E}}(\cdot, r)$ on $r$. In this case, there exists some value of $r$ such that $\hat{E}^{A}=\hat{\mathbf{E}}(\cdot, r)$. Note that continuity of $\hat{\mathbf{E}}(\cdot, r)$ is ensured by the continuity of the parametric description of the solution $\mathbf{V}_{a}(r)$. If the modes are not continuous, the existence of $\hat{E}^{A}$ is also guaranteed provided that $\mathbf{C}^{A}$ is a regular matrix. In this case, $\hat{E}^{A}$ does not necessarily coincide with any value of $\hat{\mathbf{E}}(\cdot, r)$.

In the parametric case, the error in the QoI reads

$$
E_{Q o I}=L(\mathbf{V})-L\left(\mathbf{V}_{a}\right)=\int_{r} \operatorname{diag}\left(\hat{\boldsymbol{\lambda}}_{p}(\cdot, r)^{\top} \hat{\mathbf{E}}(\cdot, r)\right) d r=\operatorname{diag}\left(\hat{\boldsymbol{\lambda}}_{p}^{\top} \hat{\mathbf{E}}\right) \mathbf{M}_{r} \mathbb{1}_{n_{r}},
$$

where the last term in the right uses the multidimensional tensor structure to express the integrals along the $r$ range by a scalar product.

An accumulated value of $\hat{\boldsymbol{\lambda}}_{p}, \hat{\lambda}^{A} \in \mathbb{R}^{2 n}$, is readily introduced

$$
\hat{\lambda}^{A}=\int_{r} \hat{\boldsymbol{\lambda}}_{p}(\cdot, r) d r=\hat{\boldsymbol{\lambda}}_{p} \mathbf{M}_{r} \mathbb{1}_{n_{r}} .
$$

In order to obtain a suitable error representation, it must be assumed that the following hypothesis is true.

Hypothesis 1. The quantity of interest $E_{Q_{o I}}$ is expressed using the accumulated value of $\hat{\boldsymbol{\lambda}}_{p}$ and the vector $\hat{E}^{A}$, that is to say,

$$
E_{Q o I}=\left(\hat{\lambda}^{A}\right)^{\top} \hat{E}^{A}
$$

This can be interpreted as a new application of the mean value theorem in (47), with the additional assumption that the average value of $\hat{E}$ is again $\hat{E}^{A}$. Actually in this case there is no a unique average value: there exist an affine space of dimension $2 n-1$ where all the possible vectors $\hat{E}^{A}$ fulfilling the equation above lie. Thus, the assumption claiming that $\hat{E}^{A}$ from equation (45) fulfils also (47) (at least approximately) is very likely to hold. This assumption is further supported by noting that the dependence on $r$ of $\underline{\mathbf{C}}$ and $\hat{\boldsymbol{\lambda}}_{p}$ is directly given by the dependence on $r$ of $\mathbf{V}_{a}$ (the matrices $\mathbf{F}, \mathbf{G}, \mathbf{A}$ and $\mathbf{B}$ and the tensors $\underline{\mathbf{A}}$ and $\underline{\mathbf{B}}$ depend on the 
solution $\mathbf{V}_{a}$ linearly). Thus, the dominant $r$ mode in $\mathbf{V}_{a}$ is going to be the dominant $r$ mode also in $\underline{\mathrm{C}}$ and $\hat{\boldsymbol{\lambda}}_{p}$ and hence $\hat{E}^{A}$ from equation (45) is expected to fulfil also (47). An error indicator is introduced in section 4.2.2 in order to numerically check the validity of Hypothesis 1 .

Hence, the dual problem in the condensed form reads

$$
\mathbf{C}^{A^{\top}} \hat{\rho}^{A}=\hat{\lambda}^{A}
$$

and the corresponding error representation is

$$
E_{Q o I}=\left(\hat{\rho}^{A}\right)^{\top} \hat{R}^{A}\left(\mathbf{V}_{a}\right) .
$$

Thus, also in the parametric form of the problem, the error in the quantity of interest can be affordably assessed by solving the condensed dual problem (48) and computing the error estimate using (49).

\subsubsection{Tolerances and stopping criteria.}

The goal-oriented error estimates defined above are used to define the stopping criteria in Algorithm 1.

Let us introduce the error indicators $\xi_{\star}^{\square}$, where $\square$ accounts for the type of error measured (taking three possible values: $\square=R$ for a purely residual estimate; $\square=S$ for a measure of the stationarity in the loop, the difference with the previous term or iteration; or $\square=Q o I$ for the error in the quantity of interest as described above), and $\star$ denotes the loop where it is used (taking three possible values: $\star=\gamma ; \star=M$; or $\star=k$ ).

Thus, the different stopping criteria are expressed as: continue with the loop while $\xi_{\star}^{\square}>\operatorname{tol}_{\star}^{\square}$, tol $_{\star}^{\square}$ being the different tolerances prescribed for the different criteria.

The definitions of the different error indicators are listed below:

1. Loop in $\gamma$

$$
\xi_{\gamma}^{R}=\frac{\left\|\hat{R}^{A}\left(\mathbf{V}_{a}^{[\gamma+1]}\right)\right\|_{2}}{\left\|\hat{S}^{A}\right\|_{2}}, \quad \xi_{\gamma}^{S}=\frac{\left\|\mathbf{V}_{a}^{[\gamma+1]}-\mathbf{V}_{a}^{[\gamma]}\right\|_{2}}{\left\|\mathbf{V}_{a}^{[\gamma+1]}\right\|_{2}} \text { and } \xi_{\gamma}^{Q o I}=\frac{\left|\left(\hat{\rho}^{A}\right)^{\top} \hat{R}^{A}\left(\mathbf{V}_{a}^{[\gamma+1]}\right)\right|}{\left|L\left(\mathbf{V}_{a}^{[\gamma+1]}\right)\right|}
$$

where $\hat{S}^{A}=\mathbf{S} \mathbf{M}_{r} \mathbb{1}_{n_{r}}$. Recall that $\hat{R}^{A}\left(\mathbf{V}_{a}\right)=\hat{\mathbf{R}}\left(\mathbf{V}_{a}\right) \mathbf{M}_{r} \mathbb{1}_{n_{r}}$ and $\|\cdot\|_{2}$ stands for either the $L^{2}$-norm or the Frobenius norm (depending on whether the argument is a vector or a matrix).

2. Loop in $M$

$$
\xi_{M}^{R}=\frac{\left\|\hat{R}_{I}^{A}\left(\mathbf{I}_{a}\right)\right\|_{2}}{\left\|\hat{S}^{A}\right\|_{2}}, \quad \xi_{M}^{S}=\frac{\left|\alpha_{I}^{M}\right|}{\left|\alpha_{I}^{1}\right|} \text { and } \xi_{M}^{Q o I}=\frac{\left|\hat{\lambda}^{\top}\left(\mathbf{V}_{a}^{[\gamma], M}-\mathbf{V}_{a}^{[\gamma], M-1}\right)\right|}{\left|L\left(\mathbf{V}_{a}^{[\gamma]}\right)\right|},
$$

where $\hat{R}_{I}^{A}\left(\mathbf{I}_{a}\right)=\left(\mathbf{S}-\mathbf{V}_{a} \odot \mathbf{I}_{a}\right) \mathbf{M}_{r} \mathbb{1}_{n_{r}}$.

3. Loop in $k$

$$
\xi_{k}^{S_{1}}=\frac{\left\|\left(V^{M}\right)^{k+1}-\left(V^{M}\right)^{k}\right\|_{2}}{\left\|\left(V^{M}\right)^{k+1}\right\|_{2}} \text { and } \xi_{k}^{S_{2}}=\frac{\left\|\left(\mathcal{R}^{M}\right)^{k+1}-\left(\mathcal{R}^{M}\right)^{k}\right\|_{2}}{\left\|\left(\mathcal{R}^{M}\right)^{k+1}\right\|_{2}} .
$$


Moreover, in order to check the stabilization of $\hat{\lambda}^{A}$ and $\hat{\rho}^{A}$, the following indicator is introduced:

$$
d_{\rho}=\frac{\left\|\rho^{A^{[\gamma+1]}}-\rho^{A[\gamma]}\right\|_{2}}{\left\|\rho^{A[\gamma+1]}\right\|_{2}} .
$$

If the value of $d_{\rho}$ is small enough, the assumption on the stability of $\hat{\rho}^{A}$ is going to be confirmed. Besides, for checking that the Hypothesis 1 holds, another indicator is introduced:

$$
e_{\hat{E}}=\frac{\left|E_{Q o I}-\left(\hat{\lambda}^{A}\right)^{\top} \hat{E}^{A}\right|}{\left|E_{Q o I}\right|} .
$$

Note that $\hat{E}^{A}$ is computed using equation (46) straightforwardly.

Similarly, the verification of the obtained solution and the corresponding losses is performed with the following error measures (with respect to a reference solution $\mathbf{V}$ ):

$$
\begin{gathered}
e_{V}=\frac{\left\|\mathbf{V}-\mathbf{V}_{a}\right\|_{2}}{\|\mathbf{V}\|_{2}} \\
e_{L}=\frac{\left\|l(\mathbf{V})-l\left(\mathbf{V}_{a}\right)\right\|_{2}}{\|l(\mathbf{V})\|_{2}} .
\end{gathered}
$$

\section{Numerical examples}

In all the following examples, we apply the Algorithm 1 to both the algebraic and parametric version of the Power Flow problem taking into account the proposed goal-oriented error estimates. Figure 1 shows the diagram of test system. The model, taken from [47], is a threephase system with different topologies and load characteristics including a simplified representation of the high-voltage system. The three-phase grid has 256 nodes (located in 3 different branches), and therefore the number of degrees of freedom is $n=3 \times 256=768$. However, the number of geometrical nodes in Figure 1 is only 155, numbered from a701 to $a 777$ (first branch), from $b 701$ to $b 746$ (second branch), from $c 701$ to $c 729$ (third branch), and three more: a700 connecting the three branches, the transformer and the slack node. The additional 101 nodes correspond to the duplication of the 101 nodes of the mesh where the DG can be located. These new 101 nodes are identified by adding an $l$, for instance $l c 701$ is the duplication of $c 701$. This duplication is necessary because each distributed generator (DG) is connected to the system through a step-up interconnection transformer allowing the switch from high-voltage to low-voltage.

Some of the main characteristics of the substation transformer are given below:

- High-voltage rating: $230 \mathrm{kV}$

- Low-voltage rating: $4.16 \mathrm{kV}$

The main objective in the above examples is to solve an optimization problem: find the optimal position and power of a distributed generator that minimizes the system losses, quantity of interest in this work. Considering the error assessment in the implementation of the solver allows one to compute the solution taking them into consideration. As a first step, we compute the solution while we evaluate it for calculating the losses and as a post-process or second step we look for the value that optimizes the problem statement. 


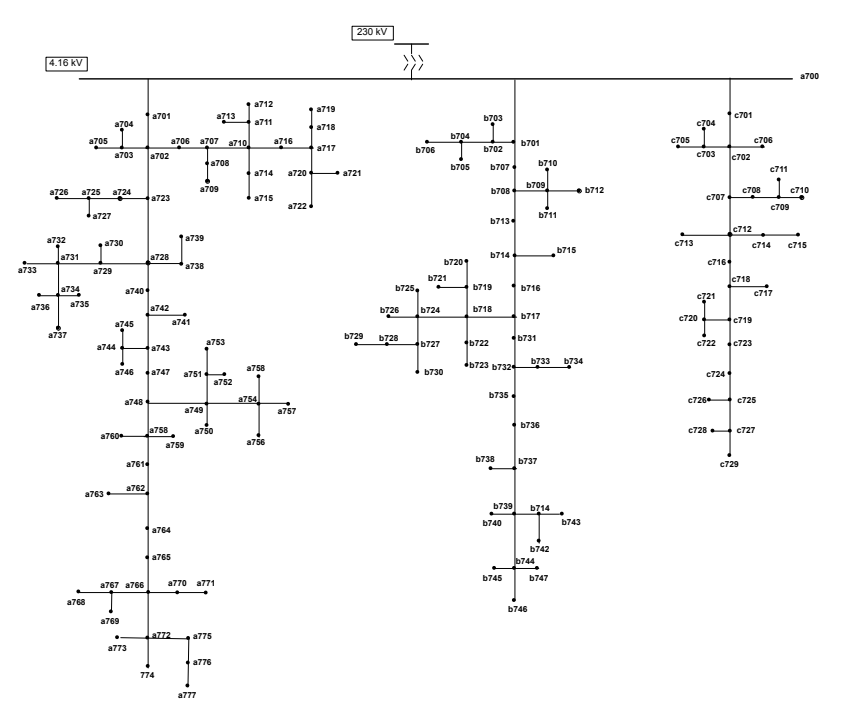

Figure 1 Diagram of the test system network

\subsection{Algebraic approach}

Our goal in this section is to show the efficiency of the procedure for linearizing the residual and the losses equation explained in section 4.1. In this case, fixing the position $q=l c 707$, the power $r=850 \mathrm{~kW}$ of the DG and the hour of the year $t=759$ we solve the power flow problem associated with these values. Consequently, $n_{q}, n_{r}, n_{t}=1$ and the number of degrees of freedom is $n=768$.

For computing the relative errors, for instance $e_{L}$, we consider as real solution the one calculated using Newton-Raphson algorithm while tolerances $\operatorname{tol}_{\gamma}^{R}$, tol ${ }_{\gamma}^{S}$ and $\operatorname{tol}_{\gamma}^{Q o I}$ are $10^{-8}$. As we mentioned above, it is not necessary to calculate the solution of the dual problem at every $\gamma$ iteration. In Figure $2 \mathrm{a}$, we observe this fact, plotting $\xi_{\gamma}^{Q o I}$ and $\xi_{\hat{\gamma}}^{Q o I}$. Note that we introduce the notation $\xi_{\hat{\gamma}}^{Q o I}$ for indicating that the vector $\hat{\rho}$ is just calculated until the tolerance for the indicator $d_{\rho}$ is reached, in this particular case that tolerance is $10^{-3}$. The standard notation $\xi_{\gamma}^{Q o I}$ implies that the dual problem is solved at every single iteration. We notice that $\hat{\rho}$ barely changes, thus the $E_{Q o I}$ does not either. In this example the stability is evident, as Figure $2 \mathrm{~b}$ shows after only 4 iterations the indicator $d_{\rho}$ verifies that $d_{\rho}<10^{-4}$, and the same fact was noticed in other simulations. That is the reason why from now on in the examples below, once we reached the tolerance for the indicator $d_{\rho}$, the vector $\hat{\rho}$ is reused in the following iterations. Hence at some point, the cost of calculating the error in the quantity of interest has the same computational cost as the residual calculation because we do not need to update $\mathbf{C}, \hat{\lambda}$ or $\hat{\rho}$. Figure 2c shows the growth of $\xi_{\gamma}^{Q o I}$ when tol ${ }_{\gamma}^{Q o I}$ increases. The numbers in the graph represent the number of iterations required until we reach the tolerance. We observe that logically this number decreases when tol ${ }_{\gamma}^{Q o I}$ increases. Moreover, in order to show the efficiency of the error indicator $\xi_{\gamma}^{Q o I}$ used as stopping criteria in the algorithm, the effectivity index is shown in Figure $2 \mathrm{~d}$ comparing the relative error with respect to the reference solution $e_{L}$ versus $\xi_{\gamma}^{Q o I}$. 


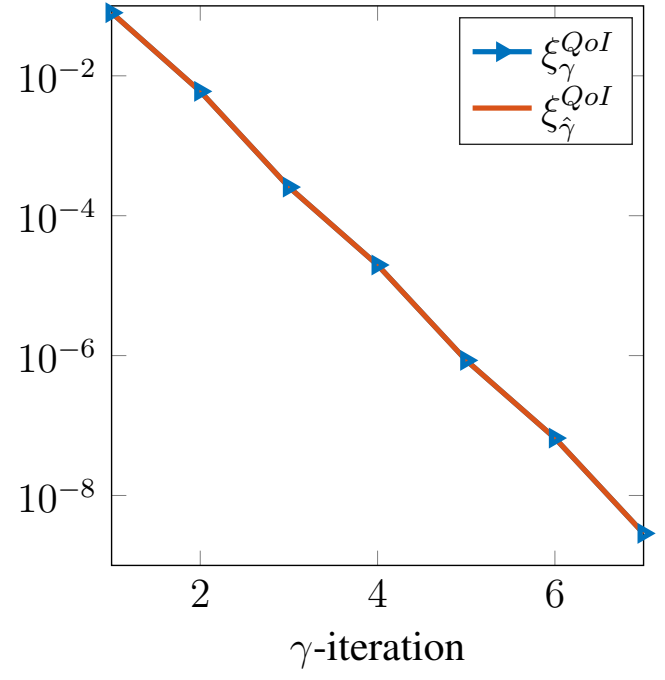

(a)

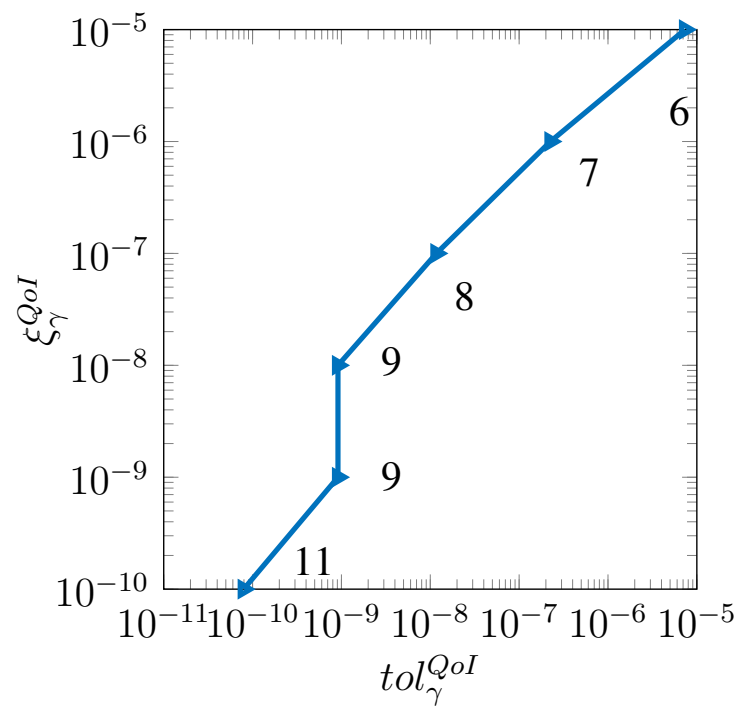

(c)

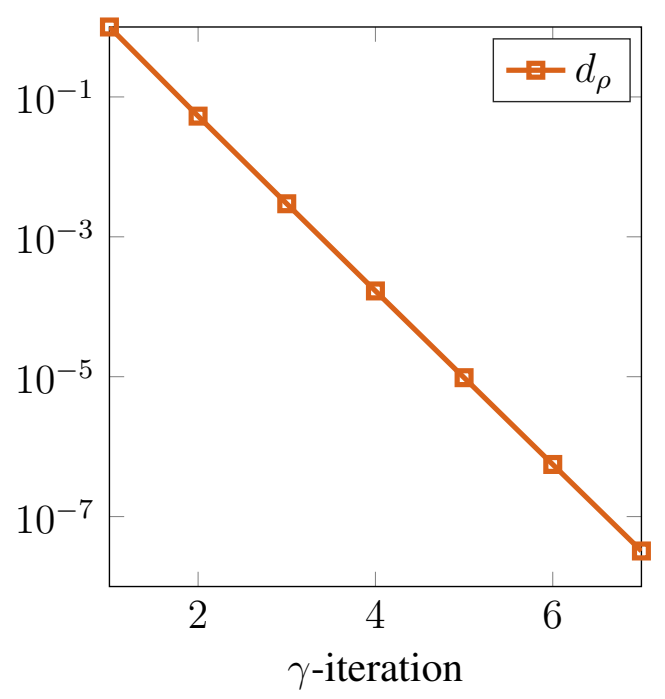

(b)

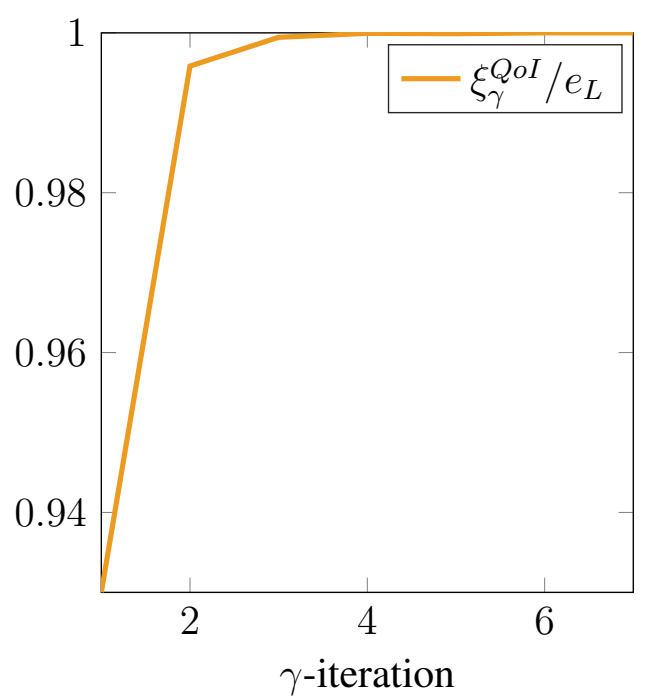

(d)

Figure 2 (a) Convergence diagram of the relative error in the losses and error in quantity of interest with the iteration index $\gamma$. (b) Convergence diagram of stagnation criteria for the solution of the dual problem $\rho$. (c) Error in the quantity of interest for different simulations varying the tolerances. The numbers reported on the curve represent number of iterations.(d) Effectivity index in the losses.

\subsection{Parametric approach}

\subsubsection{Optimal nominal power of a DG with fixed loads.}

As a first example of resolution of the parametric version of the power flow problem, the goal is to find the optimal nominal power of a DG when it is set at the node $l b 707$ (second branch) and $n_{q}, n_{t}=1$. Thus, the resolution of the Parametric Power Flow problem is shown when we consider the nominal power $r$ of the DG as a parameter. We seek the value of $r$ that minimizes the system losses. Since the loads are fixed, the problem consists in finding the voltage solution 
as a separated representation:

$$
\mathbf{V}_{a}=\sum_{m}^{M} \alpha_{V}^{m} V^{m} \otimes \mathcal{R}^{m},
$$

where $r$ belongs to a set of possible values of power that the DG can provide. That is, the partition of the interval $\left[0, r_{\max }\right]$ where the increment is $r_{\max } /\left(n_{r}-1\right)$ with $r_{\max }=800 \mathrm{~kW}$ and $n_{r}$ is the number of samples, particularly in this example $n_{r}=100$. The separated representation of the input data $\mathbf{S}$ is:

$$
\mathbf{S}=\alpha_{S}^{1} S_{1} \otimes \mathbb{1}_{n_{r}}+\alpha_{S}^{2} S_{2} \otimes r_{2}
$$

where $S_{1}$ is the vector of demand loads with dimension $n, \mathbb{1}_{n_{r}}$ is a vector of ones with dimension $n_{r}, S_{2}$ is a zero vector except for the position of the DG where 1 is placed and $r_{2}$ is a vector in $\mathbb{R}^{n_{r}}$ where $\left[r_{2}\right]_{i}=\left(i / n_{r}\right) \cdot r_{\text {max }}, \forall i=0, \ldots, n_{r}$.

The goal is to set different tolerances and compare the obtained solutions in order to validate the goal-oriented error estimates in terms of controlling the quality of the approximations. In Figure 3, the sub-figures $3 \mathrm{a}$ and $3 \mathrm{~b}$ show the stopping criteria for $\gamma$ and $M$ respectively for the first set of tolerances in the algorithm, $\operatorname{tol}_{\gamma}^{\square}=10^{-7}, \operatorname{tol}_{M}^{\square}=10^{-8}$ and $\operatorname{tol}_{k}^{\square}=10^{-10}$ for $\square=S, R, Q o I$. The numbers in sub-figure 3a represent the amount of modes that the solution contains at every iteration $\gamma$. In this case, the final solution consists of 24 modes after 9 iterations. It is remarkable that in all $M$ iterations, at some point the criterion $\xi_{M}^{R}$ stabilizes after some iterations. This might be because at every iteration $M$, we add a new term hence more information is considered. However,it is possible that the added information is not enough for changing significantly the quality of the solution, thus the residual in the first step of the algorithm does not decrease. The same quantities are shown in the other two sub-figures $5 \mathrm{a}$ and $5 \mathrm{~b}$ but the fixed tolerances are $\operatorname{tol}_{\gamma}^{\square}=10^{-5}$, $\operatorname{tol}_{M}^{\square}=10^{-6}$ and tol ${ }_{k}^{\square}=10^{-7}$ for $\square=S, R, Q o I$ in this case. As we can see, the amount of terms of the solution changes, we need just 19 in this simulation.

The introduction of error estimators in the Algorithm 1 allows to control the whole procedure and specifically the construction of the solution $\mathrm{V}$. By adding more modes to the final solution, its accuracy can increase when we compare it to the solution obtained using the algebraic version of the algorithm (it is used as reference solution). In Figure $3 \mathrm{c}$ and $5 \mathrm{c}$ this fact is observed, the accuracy of the solution and the losses improve when the tolerances are lower because the solution has more modes, i.e. 24 versus 19, thus it is more precise.

Besides, the effectivity index is shown in Figure 4a compared to the relative error in losses $e_{L}$ and the error indicator of the quantity of interest $\xi_{\gamma}^{Q o I}$. In Figure $4 \mathrm{~b}$, the proof that the Hypothesis 1 holds in the numerical examples is shown using the indicator $e_{\hat{E}}$. At every $\gamma$ iteration, the difference between the two average vectors $\hat{E}^{A}$ is insignificant.

Once we have the solution $\mathbf{V}_{a}$, the losses are calculated using the operator $L$. The minimum loss is $212 \mathrm{~kW}$ given when the power of the DG is $r=307 \mathrm{~kW}$. Table 1 shows the comparison between the solution that we get using the parametric and the algebraic version of Algorithm 1 and NR solver. 


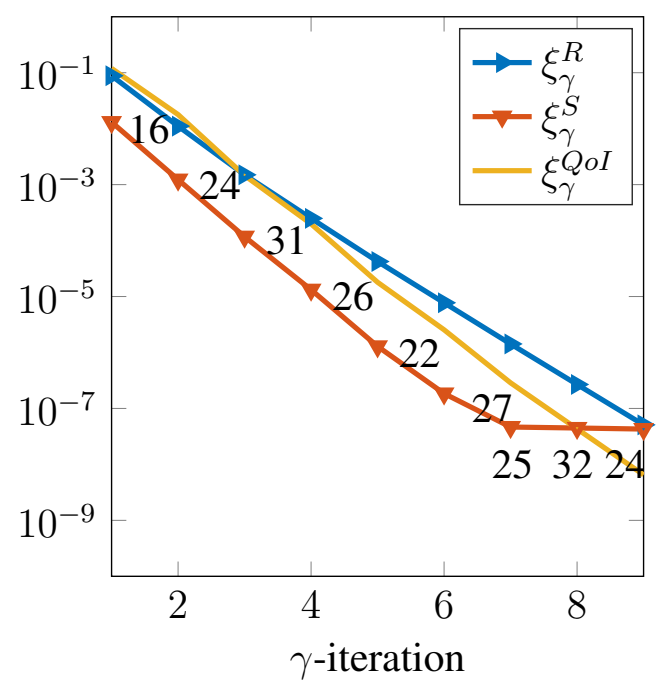

(a)

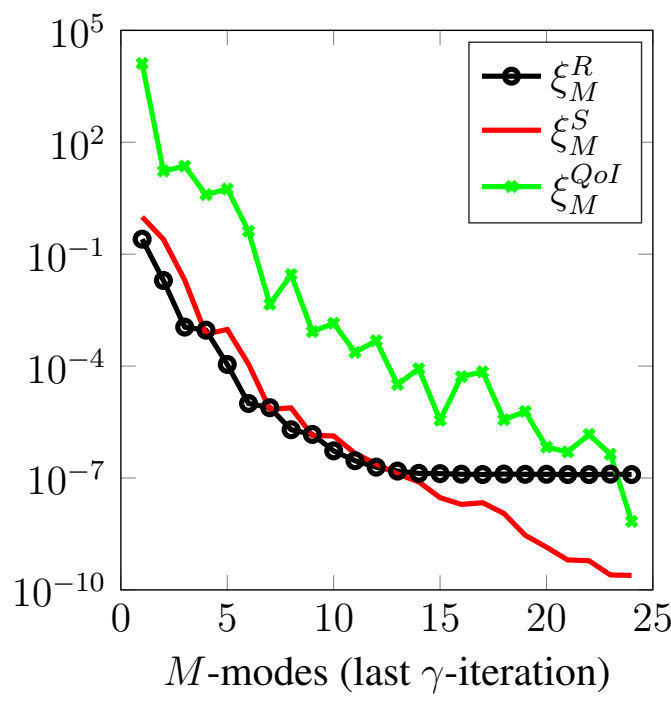

(b)

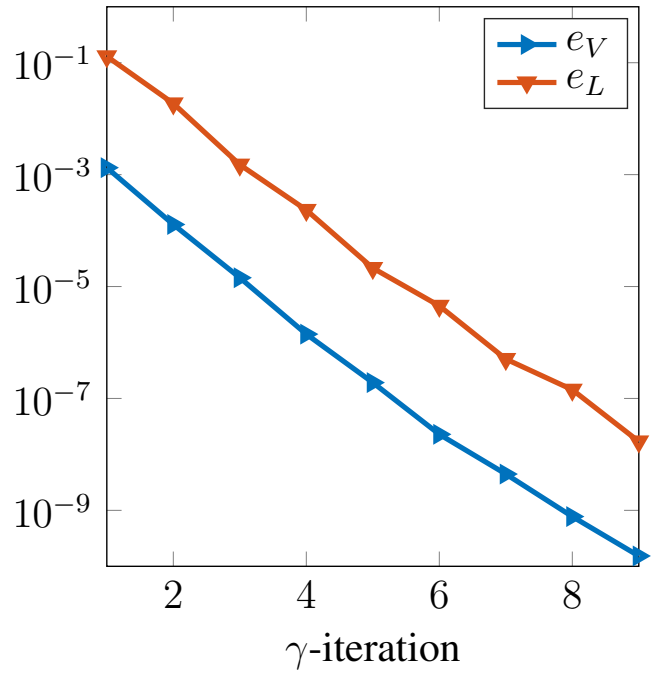

(c)

Figure 3 Diagrams of convergence for tolerances $\operatorname{tol}_{\gamma}^{\square}=10^{-7}, \operatorname{tol}_{M}^{\square}=10^{-8}$ and $\operatorname{tol}_{k}^{\square}=$ $10^{-10}$. (a) Convergence diagram of the stopping criteria for the outer loop with the iteration index $\gamma$. The numbers along the curves refer to the number of modes that the solution contains. (b) Convergence diagram of the enrichment algorithm in the last $M$ iteration (last $\gamma$-iteration). (c) Relative errors comparing the real and the approximated solution.

\subsubsection{Optimal nominal power and position of a DG with fixed loads.}

In this second example, we introduce the position of the DG $q$ as a parameter. In such manner, the goal is to seek the optimal nominal power and the position of the DG that minimizes the system losses. One more time, the range of values that $r$ can take is the partition of the interval $\left[0, r_{\max }\right]$ when the increment is the same as before, $r_{\max } /\left(n_{r}-1\right)$, with $n_{r}=100$ and $r_{\max }=$ $800 \mathrm{~kW}$. We set the DG in the two first branches corresponding with $q=1, \ldots, 82$ being $n_{q}=82$ while $n_{t}=1$.

Following the same procedure as before, we fix two shorts list of tolerances. In the first case, 


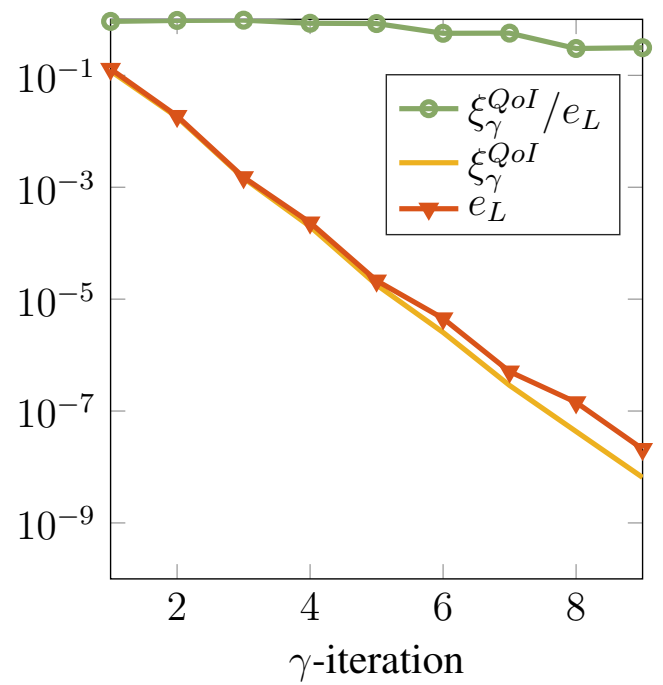

(a)

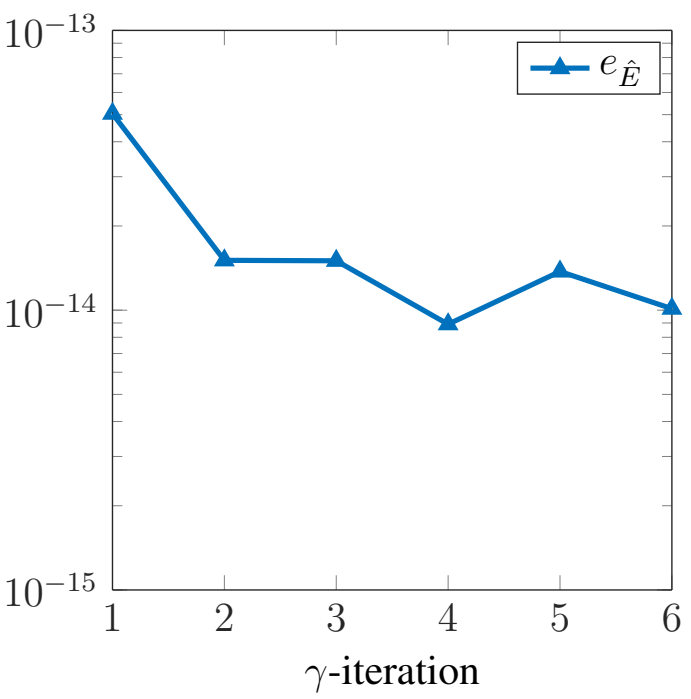

(b)

Figure 4 (a) Effectivity index in the losses.(b) Convergence diagram of the error indicator $e_{\hat{E}}$ with the iteration index $\gamma$.

\begin{tabular}{|c|c|c|c|c|}
\hline Method & Par. Version (case 1) & Par. Version (case 2) & Alg. Version & NR \\
\hline Minimum Losses $(\mathrm{kW})$ & 212.126925 & 212.126518 & 212.126921 & 212.126925 \\
\hline Optimal power $r(\mathrm{~kW})$ & 307 & 307 & 307 & 307 \\
\hline
\end{tabular}

Table 1 Comparative table showing the optimal value of $r$ and the minimum losses when the solution is calculated using different numerical methods.

the tolerances are $\operatorname{tol}_{\gamma}^{\square}=10^{-7}, \operatorname{tol}_{M}^{\square}=10^{-8}$ and $\operatorname{tol}_{k}^{\square}=10^{-10}$ for $\square=S, R, Q o I$. Figure 6 shows the diagrams of convergence for $\gamma$ and the last $M$ iteration. The final solution is composed of 29 terms. The criterion $\xi_{M}^{R}$ stabilizes after a few iterations. Similarly, when the tolerances are $\operatorname{tol}_{\gamma}^{\square}=10^{-5}$, $\operatorname{tol}_{M}^{\square}=10^{-6}$ and $\operatorname{tol}_{k}^{\square}=10^{-7}$ for $\square=S, R, Q o I$, Figure 7 shows how the stopping criteria are reached. In this case, the number of terms is lower, 24 and only 9 $\gamma$ iterations are needed. It is worth mentioning that even when the two approximations have a different number of terms for accuracy, the difference is hardly noticeable, see Figures $6 \mathrm{c}$ and $7 \mathrm{c}$.

As a post-process the evaluation of the system losses is carried out evaluating the operator $L$. The two dimensional representation of the losses is presented in Figure 8 where we can observe that the minimum value of the losses corresponds to a DG situated in the second branch. Concretely, the minimum loss is $193 \mathrm{~kW}$ when the DG is set at the position $l b 736$ with power $573 \mathrm{~kW}$. Note that the process of optimization could be carried out with any algorithm, since the objective function is now explicitly available. 


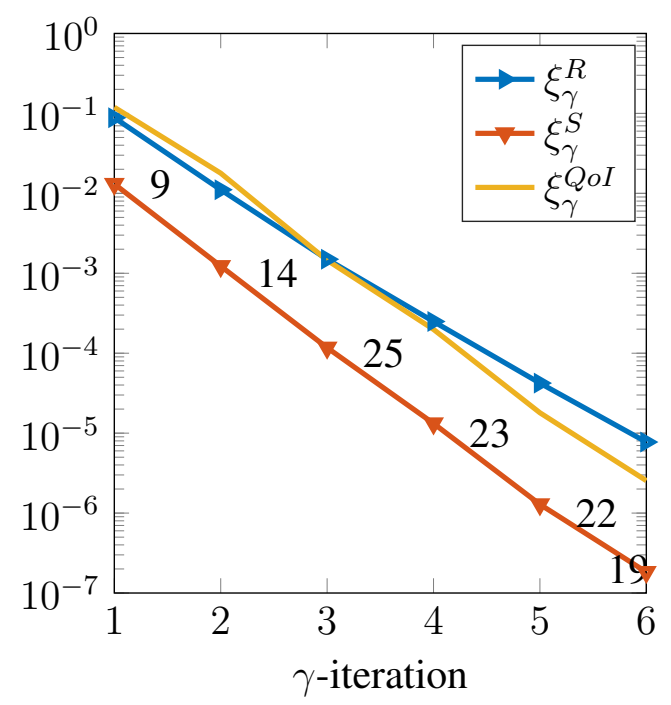

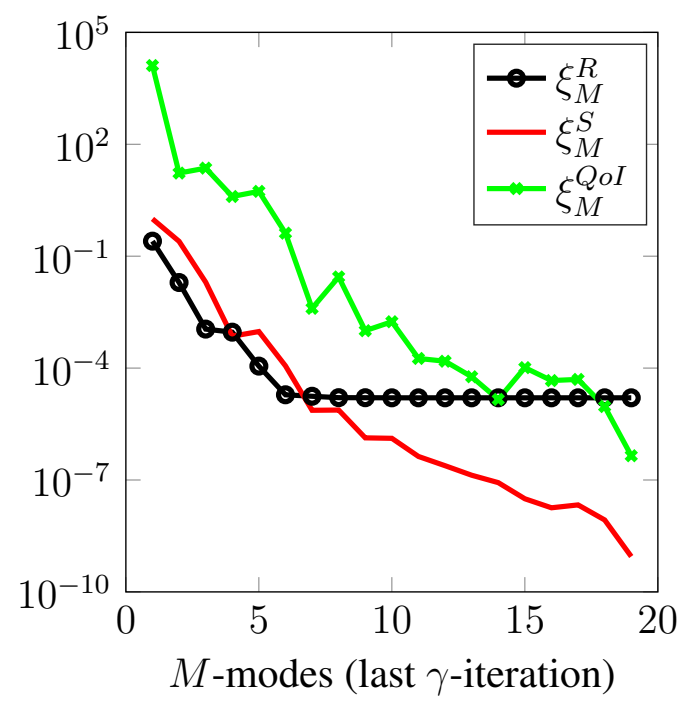

(b)

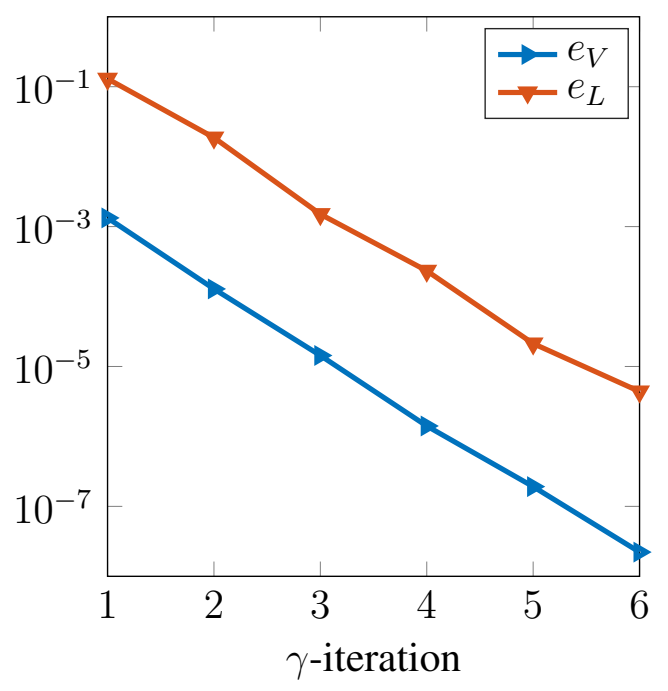

(c)

Figure 5 Diagrams of convergence for tolerances tol ${ }_{\gamma}^{\square}=10^{-5}$, tol $_{M}^{\square}=10^{-6}$ and tol ${ }_{k}^{\square}=10^{-7}$.

(a) Convergence diagram of the stopping criteria for the outer loop with the iteration index $\gamma$. The numbers along the curves refer to the number of modes that the solution contains. (b) Convergence diagram of the enrichment algorithm in the last $M$ iteration (last $\gamma$-iteration). (c) Relative errors comparing the real and the approximated solution.

\subsubsection{Optimal positioning of a DG with time varying loads.}

The goal in this last example is to seek the optimal nominal and position of the DG over a year when the parameter time $t$ is introduced. Now, we consider three DGs, each of them is set in a different branch. The parameters associated to this problem are the position of the DGs $q_{1}$, $q_{2}$ and $q_{3}$, the active power of the DGs $r_{1}, r_{2}$ and $r_{3}$ and the time $t$. The representation of the 


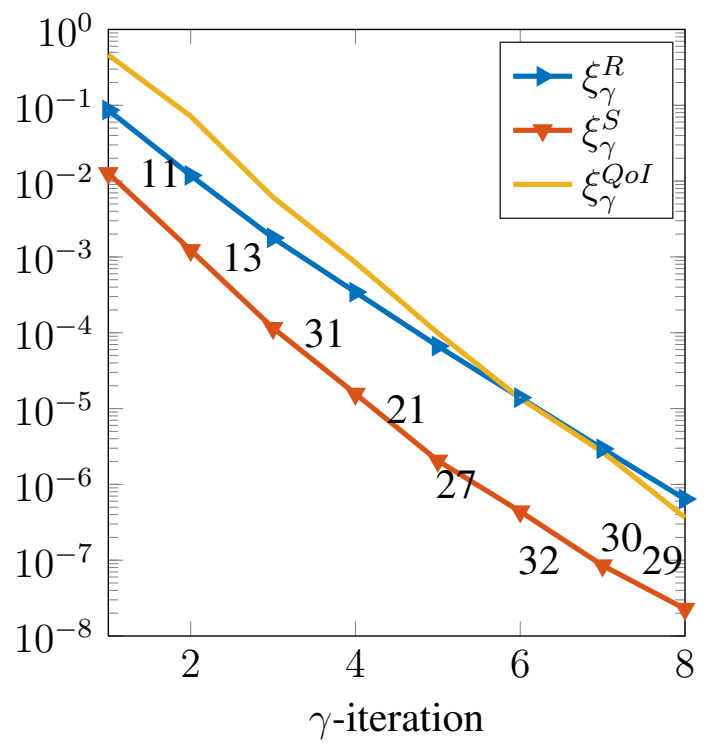

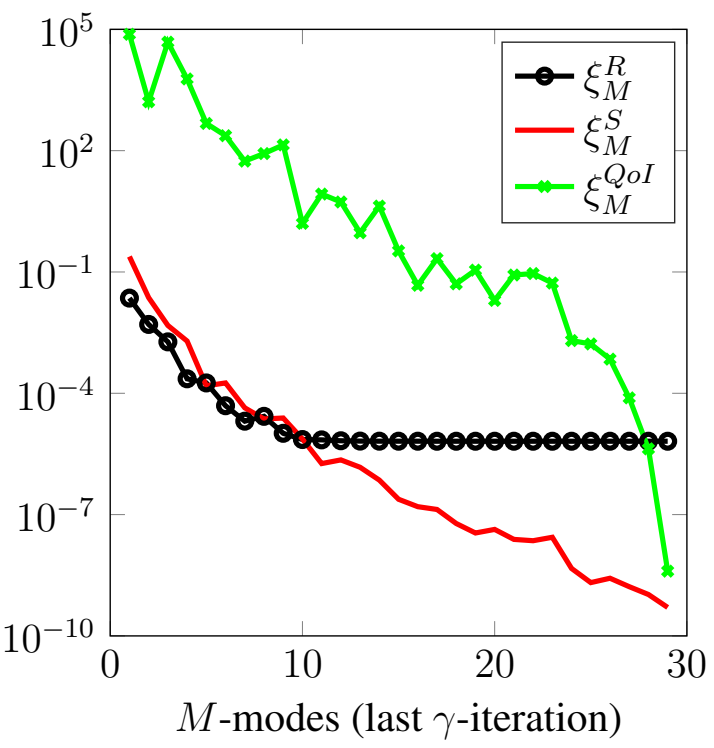

(b)

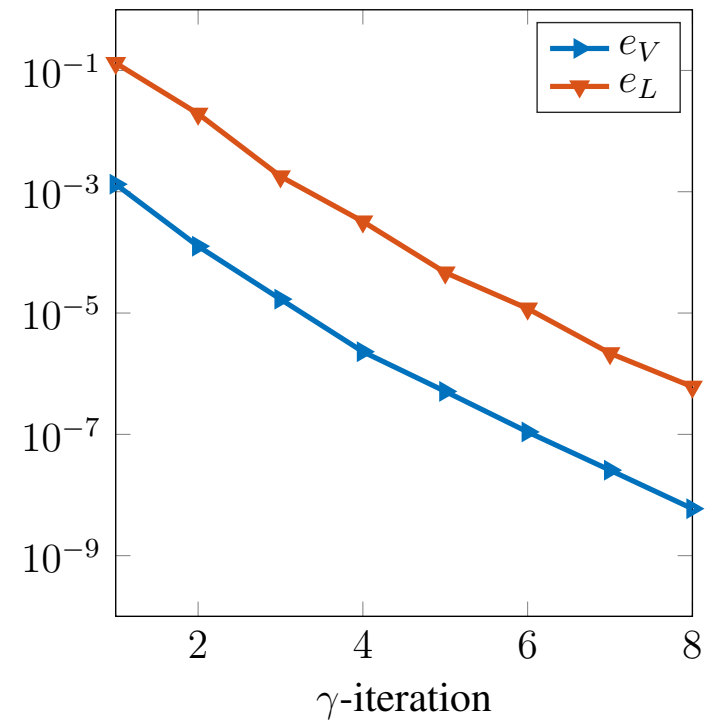

(c)

Figure 6 Diagrams of convergence when the tolerances are tol ${ }_{\gamma}^{\square}=10^{-7}$, tol $_{M}^{\square}=10^{-8}$ and $\operatorname{tol}_{k}^{\square}=10^{-10}$. (a) Convergence diagram of the stopping criteria for the outer loop with the iteration index $\gamma$. The numbers along the curves refer to the number of modes that the solution contains. (b) Convergence diagram of the enrichment algorithm in the last $M$ iteration (last $\gamma$-iteration). (c) Relative errors comparing the real and the approximated solution.

solution is given by

$$
\mathbf{V}_{a}=\sum_{m}^{M} \alpha_{V}^{m} V^{m} \otimes \mathcal{Q}^{m} \otimes \mathcal{R}^{m} \otimes \mathcal{T}^{m}
$$




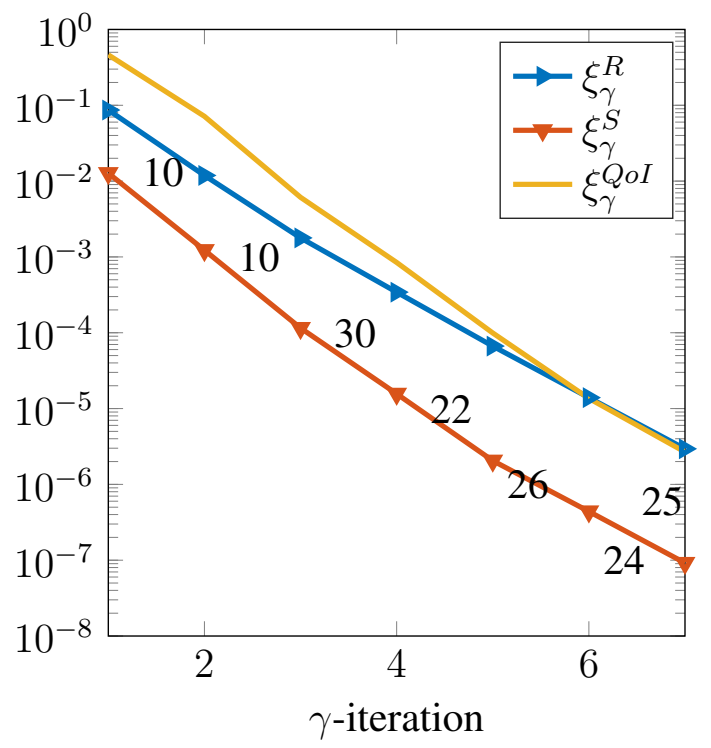

(a)

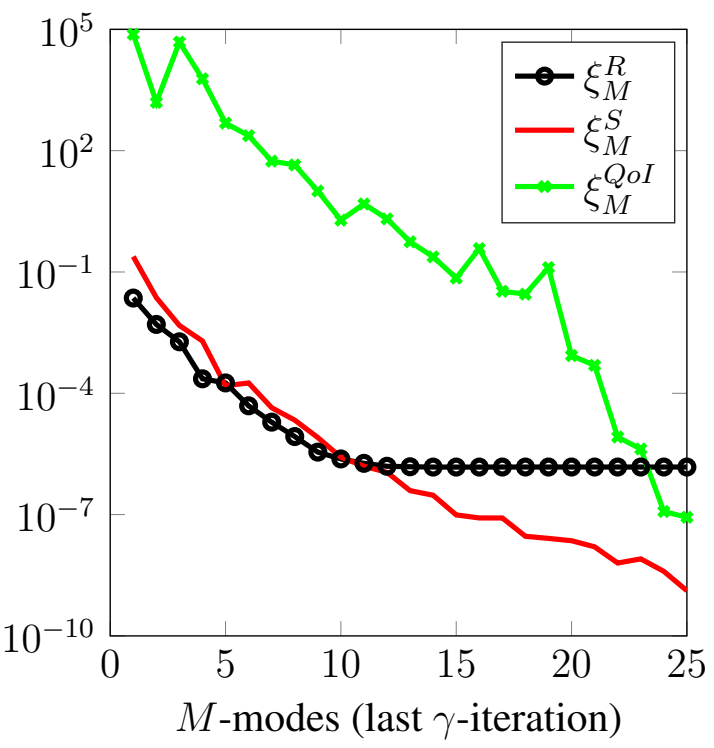

(b)

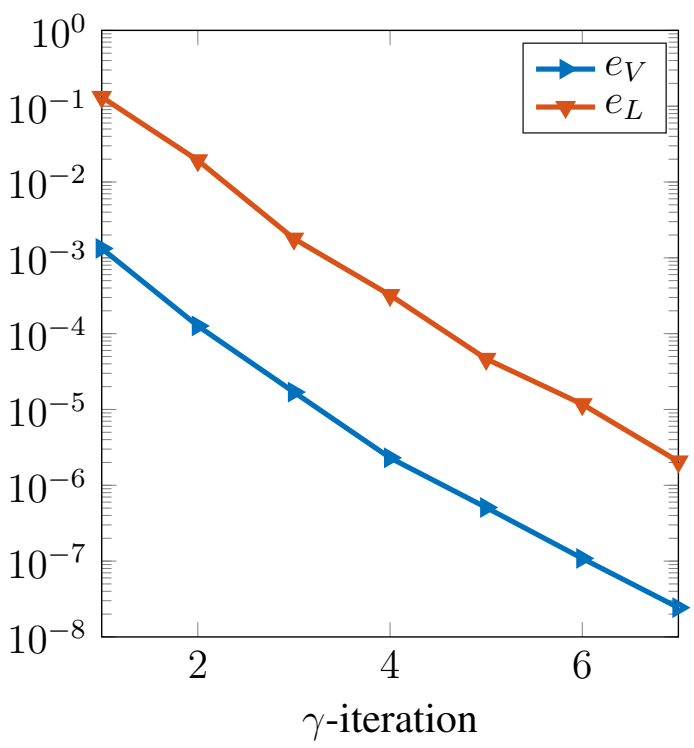

(c)

Figure 7 Diagrams of convergence when the tolerances are tol ${ }_{\gamma}^{\square}=10^{-5}$, $\operatorname{tol}_{M}^{\square}=10^{-6}$ and $\operatorname{tol}_{k}^{\square}=10^{-7}$. (a) Convergence diagram of the stopping criteria for the outer loop with the iteration index $\gamma$. The numbers along the curves refer to the number of modes that the solution contains. (b) Convergence diagram of the enrichment algorithm in the last $M$ iteration (last $\gamma$-iteration). (c) Relative errors comparing the real and the approximated solution.

while the representation of the load term $\mathbf{S}$ requires more terms:

$$
\begin{array}{r}
\mathbf{S}=\sum_{h=1}^{24} \alpha_{S}^{h} \breve{S}^{h} \otimes \breve{Q}^{h} \otimes \breve{\mathcal{R}}^{h} \otimes \breve{\mathcal{T}}^{h}+\alpha^{1} S^{1} \otimes \mathcal{Q}^{1} \otimes \mathcal{R}^{1} \otimes \mathcal{T}^{1}+\alpha^{2} S^{2} \otimes \mathcal{Q}^{2} \otimes \mathcal{R}^{2} \otimes \mathcal{T}^{2}+ \\
\alpha^{3} S^{3} \otimes \mathcal{Q}^{3} \otimes \mathcal{R}^{3} \otimes \mathcal{T}^{3}
\end{array}
$$




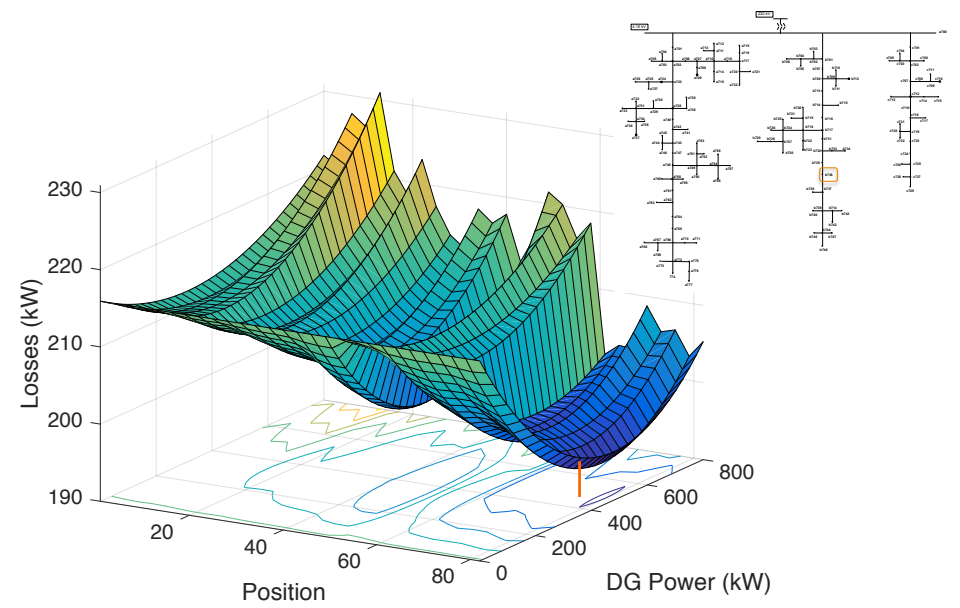

Figure 8 Reconstructed System Losses

where $\forall i=1,2,3 S^{i}$ are $n$ vectors representing the nodal positions of the network, $\mathcal{Q}^{i}$ are zero vectors of $n_{q}$ components except for the position of the DGs varying from 1 to 19 and $\mathcal{R}^{i}$ corresponds to the variation of the power. Due to the characteristics of the first two branches, the variation of $r_{1}$ and $r_{2}$ is the same with $r_{\max }=400 \mathrm{~kW}$, while the maximum value for $r_{3}$ is $2200 \mathrm{~kW}, n_{r}$ is also equal to 100 . The functions $\mathcal{T}^{i}$ expresses the load curves that represent the DGs for every hour of the year. Apart from these three terms, the load demand and the generation profiles during a year are represented by the load curves $\breve{\mathcal{T}}^{h}(t), \forall h=1, \ldots, 24$. For this particular system, 24 load curves were generated using the software HOMER described in [48]. These load curves are mainly based on solar and wind data but also depend on the customer type (commercial, industrial or residential). The time parameter $t$ is varied from 1 to 8760 with a time step of $1 \mathrm{~h}$, thus $n_{t}=8760$.

Due to the separated representation of the input source $\mathbf{S}$ containing all the information about the load during one year, more $\gamma$ iterations are necessary for reaching the tolerances in both cases, 15 and 9 iterations, as can be seen in Figures 9a and 10a. Accordingly, the number of modes of the final solution also varies, 52 versus 41 modes. The diagrams of the relative errors shown in Figures 9c and 10c point out that the solution in the first case is more accurate than the second.

The reconstruction of the losses is shown in Figure 11 where the positions of the DGs la724, lb726 and $l c 726$ that provided the annual optimal losses $341 \mathrm{~kW}$ can be seen. In terms of the parameters, the values that provide this minimal loss are $r_{1}=121 \mathrm{~kW}, r_{2}=365 \mathrm{~kW}$ and $r_{3}=311$ $\mathrm{kW}$.

\section{Conclusions}

The paper introduces a goal-oriented error estimation strategy for the Parametric Power Flow problem. The estimate is defined in the context of a PGD nonlinear solver. The error is assessed using a residual error representation and it is used to obtain consistent stopping criteria for all the iterative schemes of the PGD algorithm. 


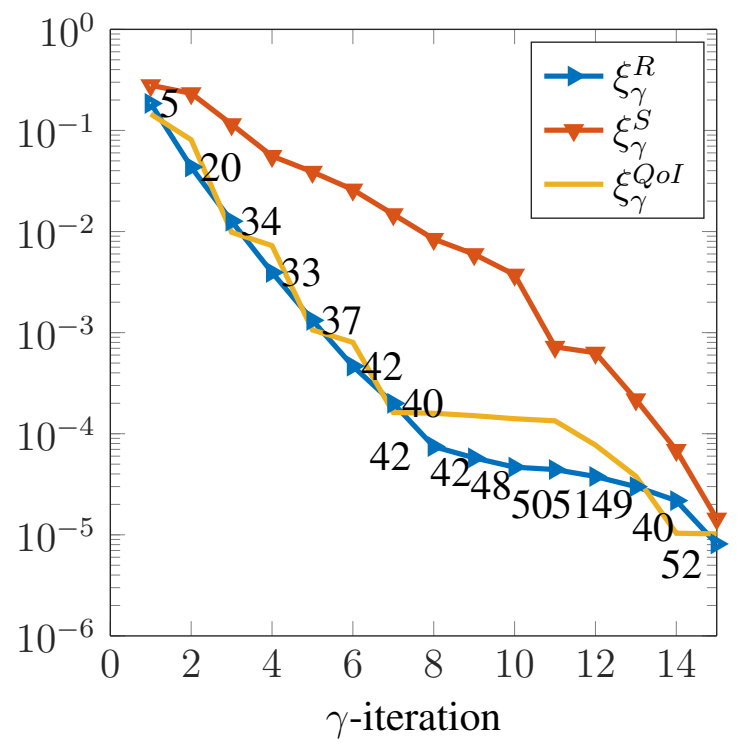

(a)

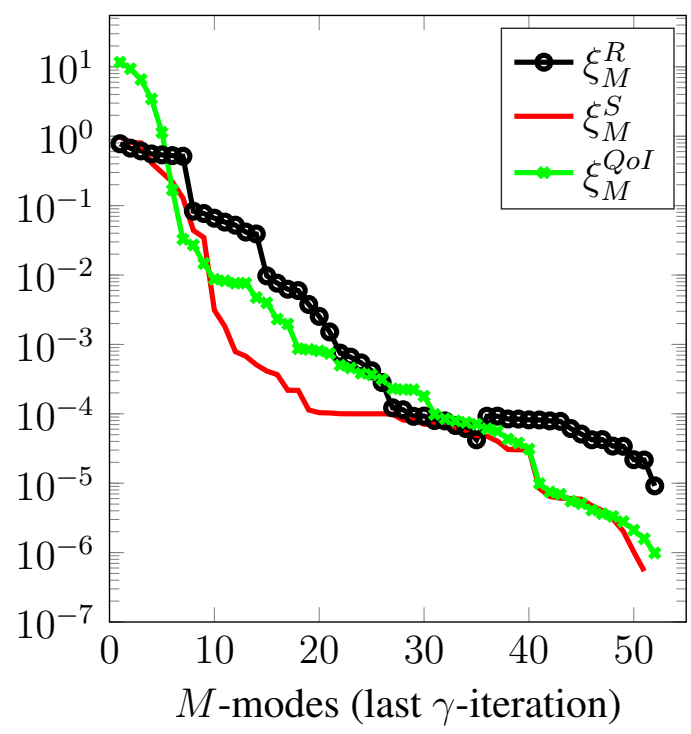

(b)

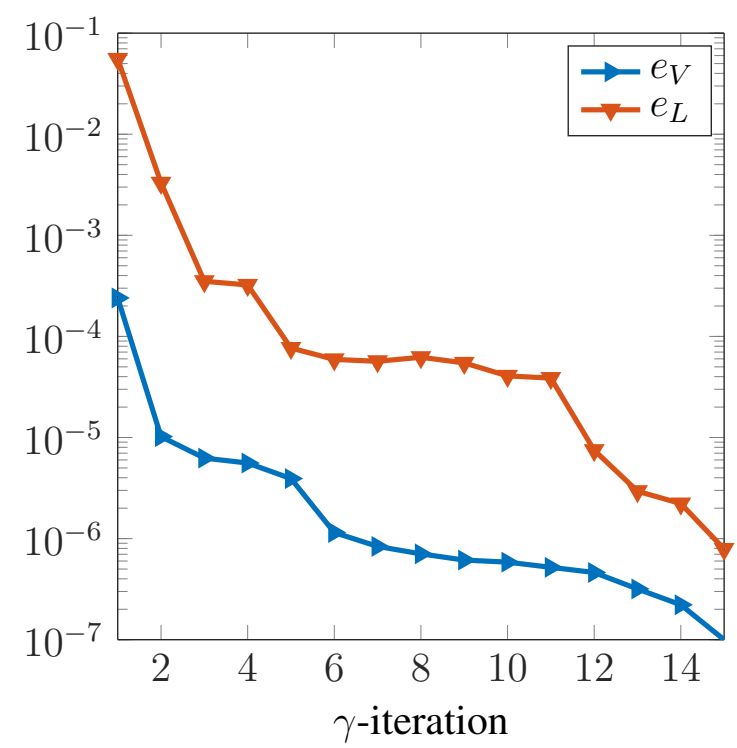

(c)

Figure 9 Diagrams of convergence when the tolerances are tol ${ }_{\gamma}^{\square}=10^{-5}$, $\operatorname{tol}_{M}^{\square}=10^{-6}$ and $\operatorname{tol}_{k}^{\square}=10^{-7}$. (a) Convergence diagram of the stopping criteria for the outer loop with the iteration index $\gamma$. The numbers along the curves refer to the number of modes that the solution contains. (b)Convergence diagram of the enrichment algorithm in the last $M$ iteration (last $\gamma$-iteration). (c) Relative errors comparing the real and the approximated solution.

The Parametric Power Flow problem is nonlinear and has complex unknowns. The challenges encountered in the derivation of the error representation and the definition of the linearized error equations arise from these two difficulties. The linearization requires not only neglecting the quadratic terms but also using a Cartesian representation.

Although the adjoint (or dual) problem depends on the solution itself, in practice it is observed 


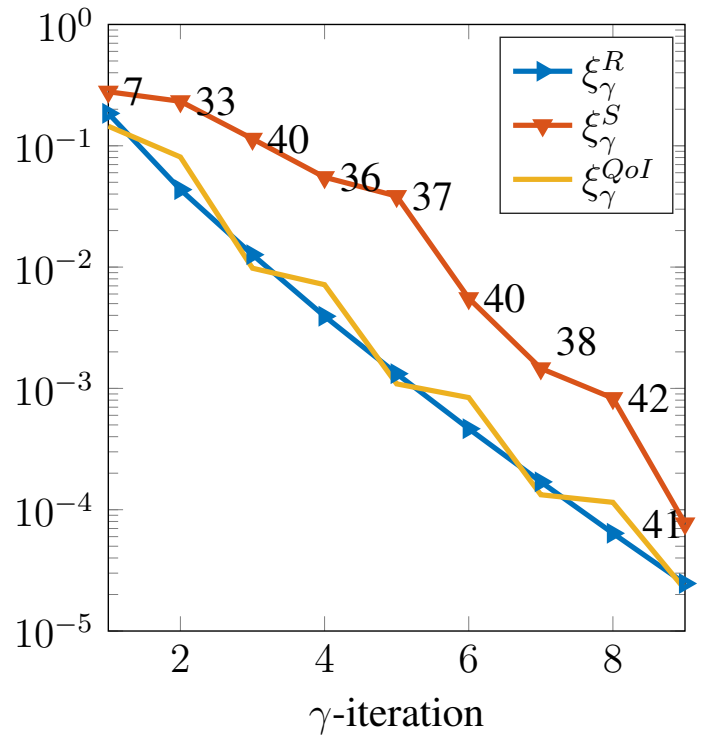

(a)

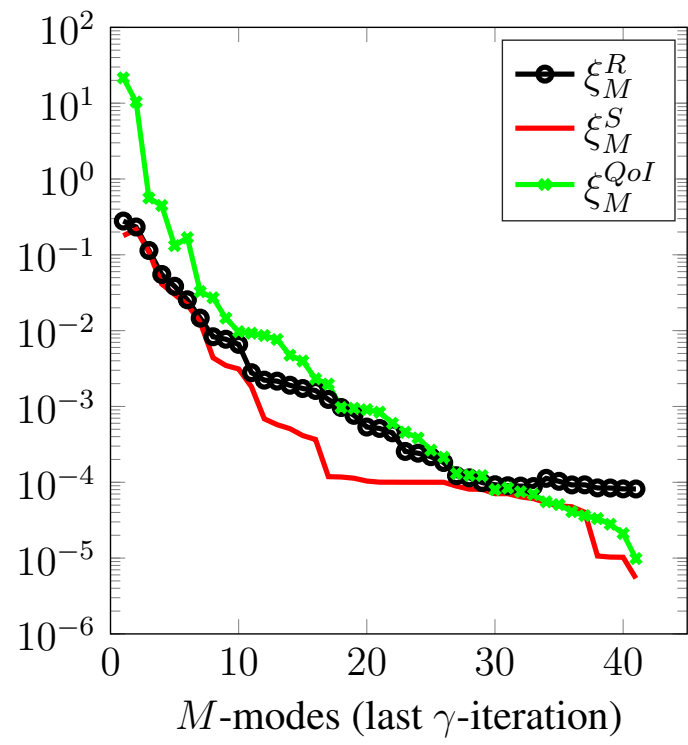

(b)

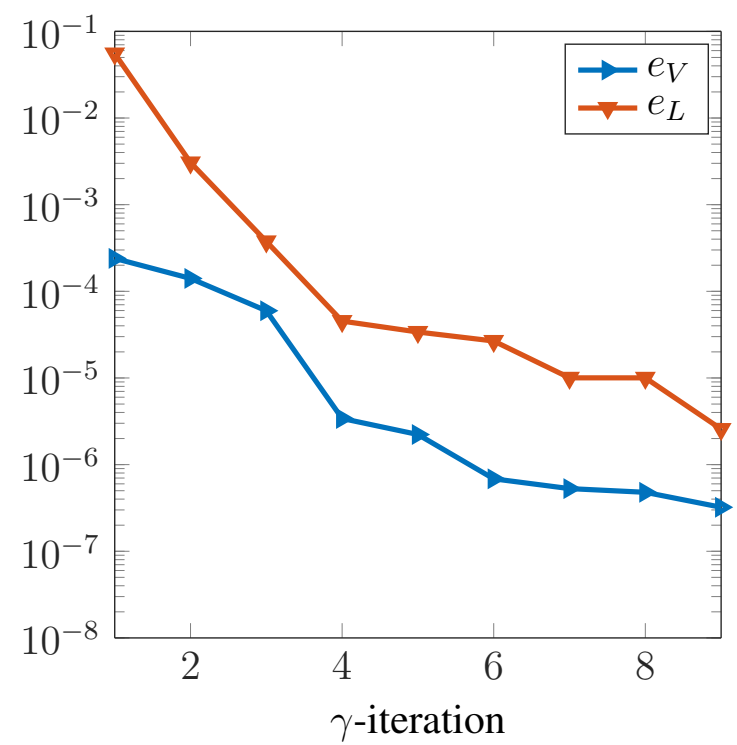

(c)

Figure 10 Diagrams of convergence when the tolerances are tol ${ }_{\gamma}^{\square}=10^{-4}, \operatorname{tol}_{M}^{\square}=10^{-5}$ and $\operatorname{tol}_{k}^{\square}=10^{-6}$. (a) Convergence diagram of the stopping criteria for the outer loop with the iteration index $\gamma$. The numbers along the curves refer to the number of modes that the solution contains. (b) Convergence diagram of the enrichment algorithm in the last $M$ iteration (last $\gamma$-iteration). (c) Relative errors comparing the real and the approximated solution.

to be stationary along the iterative process. This saves a number of computational resources because the adjoint problem is solved just once.

The condensation of the parametric dimensions is also a key aspect to make the proposed technique computationally affordable. To properly define a condensed version of the error equation and the error representation, an additional hypothesis has to be formulated stating the 


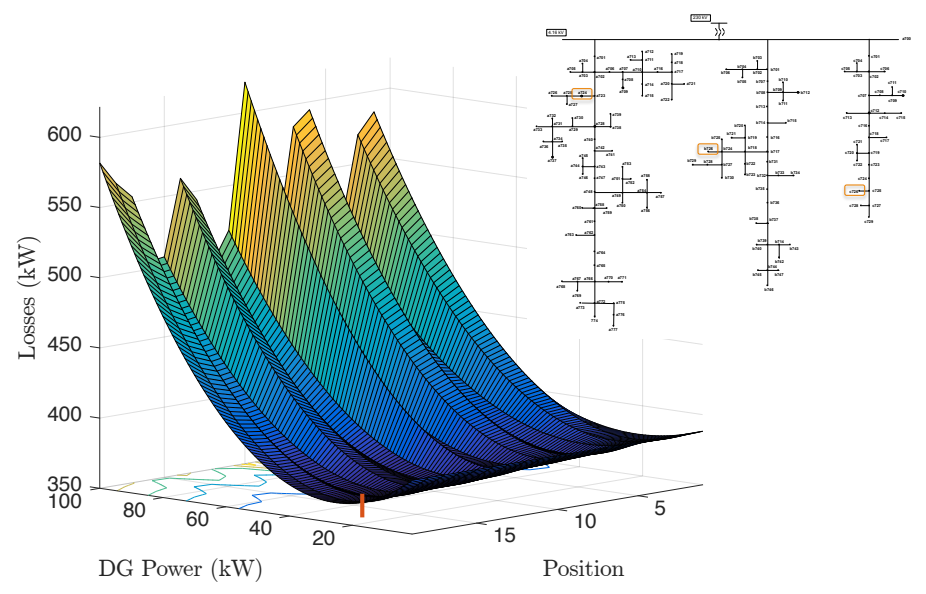

Figure 11 Reconstructed System Losses

coincidence of two mean values of the parametric error. In practice, this hypothesis is fulfilled with a very good accuracy.

This new computational strategy is used in the framework of optimal allocation of Distributed Generators in a grid, based on losses minimization. In the context of the optimization iterative techniques, the nonlinear PGD solver provides multiple queries of the model with the prescribed accuracy at very low computational cost. 


\section{References}

[1] Tinney WF, Hart CE. Power Flow Solution by Newton's Method. IEEE Transactions on Power Apparatus and Systems, 1967; (11).

[2] Wasley R, Shlash M. Newton-Raphson algorithm for 3-phase load flow. Proceedings of the Institution of Electrical Engineers, 7, 1974.

[3] Huang G, Ongsakul W. An adaptive SOR algorithm and its parallel implementation for power system applications. Parallel and Distributed Processing, 1994. Proceedings. Sixth IEEE Symposium on, 1994; 84-91.

[4] Teng JH. A modified Gauss-Seidel algorithm of three-phase power flow analysis in distribution networks. International Journal of Electrical Power \& Energy Systems 2002; 24(2):97-102.

[5] Stott B, Alsac O. Fast Decoupled Load Flow. Power Apparatus and Systems, IEEE Transactions on May 1974; PAS-93(3):859-869.

[6] Wu F. Theoretical study of the convergence of the fast decoupled load flow. Power Apparatus and Systems, IEEE Transactions on 1977; 96(1):268-275.

[7] Glimn A, Stagg G. Automatic calculation of load flows. Power apparatus and systems, part iii. transactions of the american institute of electrical engineers 1957; 76(3):817825.

[8] Hale H, Goodrich R. Digital computation or power flow - some new aspects. Power Apparatus and Systems, Part III. Transactions of the American Institute of Electrical Engineers 1959; 78(3):919-923.

[9] Gupta P, Humphrey Davies M. Digital computers in power system analysis. Proceedings of the IEEE-Part A: Power Engineering 1961; 108(41):383-398.

[10] Brameller A, Denmead J. Some improved methods for digital network analysis. Proceedings of the IEEE-Part A: Power Engineering 1962; 109(43):109-116.

[11] Brown H, Carter G, Happ H, Person C. Power flow solution by impedance matrix iterative method. Power Apparatus and Systems, IEEE Transactions on 1963; 82(65):1-10.

[12] Benato R, Paolucci A, Turri R. Power flow solution by a complex admittance matrix method. European Transactions on Electrical Power 2001; 11(3):181-188.

[13] Chen TH, Chen MS, Hwang KJ, Kotas P, Chebli E. Distribution system power flow analysis-a rigid approach. IEEE Trans. Power Del. 1991; 6(3):1146-1152.

[14] Gómez-Expósito A, Conejo AJ, Cañizares C. Electric energy systems: analysis and operation. CRC Press, 2008.

[15] Chen Y, Shen C. A Jacobian-free Newton-GMRES (m) method with adaptive preconditioner and its application for power flow calculations. Power Systems, IEEE Transactions on 2006; 21(3):1096-1103. 
[16] Idema R, Lahaye DJ, Vuik C, Van der Sluis L. Scalable Newton-Krylov solver for very large power flow problems. Power Systems, IEEE Transactions on 2012; 27(1):390-396.

[17] Cheng CFZ. A modified Newton method for radial distribution system power flow analysis. IEEE Transactions on Power Systems 1997; 12(1):389-397.

[18] De Leon F, Semlyen A. Iterative solvers in the Newton power flow problem: preconditioners, inexact solutions, and partial jacobian updates. IEEE Proceedings-Generation, Transmission and Distribution 2002; 149(4):479-484.

[19] Monticelli A, Garcia A, Saavedra O. Fast decoupled load flow: hypothesis, derivations, and testing. Power Systems, IEEE Transactions on 1990; 5(4):1425-1431.

[20] Lin WM, Teng JH. Three-phase distribution network fast-decoupled power flow solutions. International Journal of Electrical Power \& Energy Systems Jun 2000; 22(5):375-380.

[21] Costa VD, Pereira J, Martins N. An augmented Newton-Raphson power flow formulation based on current injections. International Journal of Electrical Power \& Energy Systems 2001; 23(4):305 - 312.

[22] Yi-Shan Z, Hsiao-Dong C. Fast Newton-FGMRES solver for large-scale power flow study. Power Systems, IEEE Transactions on May 2010; 25(2):769-776.

[23] Trias A. The holomorphic embedding load flow method. Power and Energy Society General Meeting, 2012 IEEE, 2012; 1-8.

[24] Sauer P. Explicit load flow series and functions. IEEE Trans. on Power App. and Syst. Aug 1981; PAS-100(8):3754-3763.

[25] Xu W, Liu Y, Salmon J, Le T, Chang G. Series load flow: a novel noniterative load flow method. IEE Proc.-Gener. Transm. Distrib. May 1998; 145(3):251-256.

[26] Borzacchiello D, Malik M, Chinesta F, García-Blanco R, Diez P. Unified formulation of a family of iterative solvers for power systems analysis. to appear in Electric Power Systems Research 2016; .

[27] García-Blanco R, Borzacchiello D, Chinesta F, Diez P. A reduced order modeling approach for optimal allocation of distributed generation in power distribution systems. Energy Conference (ENERGYCON), 2016 IEEE International, 2016.

[28] Borzacchiello D, Chinesta F, García-Blanco R, Diez P. Introduction to the proper generalized decomposition for the solution of the parametrized power equations. Submitted 2016; .

[29] Ammar A, Chinesta F, Diez P, Huerta A. An error estimator for separated representations of highly multidimensional models. Computer Methods in Applied Mechanics and Engineering 2010; 199(25-28):1872 - 1880.

[30] Ladevèze $\mathrm{P}$, Chamoin L. On the verification of model reduction methods based on the proper generalized decomposition. Computer Methods in Applied Mechanics and Engineering 2011; 200(23-24):2032 - 2047. 
[31] Dimitrovski A, Tomsovic K. Slack bus treatment in load flow solutions with uncertain nodal powers. Probabilistic Methods Applied to Power Systems, 2004 International Conference on, 2004; 532-537.

[32] Grainer JJ, Stevenson W. Power System Analysis. 2008.

[33] S M L Kabir MR A H Chowdhury, Alam J. Inclusion of slack bus in newton raphson load flow study. Electrical and Computer Engineering (ICECE), 2014 International Conference on, Dhaka 2014; :282-284.

[34] Ladevèze P, Simmonds J. Nonlinear Computational Structural Mechanics: New Approaches and Non-Incremental Methods of Calculation. Mechanical Engineering Series, Springer New York, 1999.

[35] Lin G, Elizondo M, Lu S, Wan X. Uncertainty quantification in dynamic simulations of large-scale power system models using the high-order probabilistic collocation method on sparse grids. Int. J. Uncert. Quant. 2014; 4(3):185-204.

[36] Zhang Z, Nguyen HD, Turitsyn K, Daniel L. Probabilistic power flow computation via low-rank and sparse tensor recovery. IEEE Trans. Power Systems 2015; .

[37] Zhang H, Li P. Application of sparse-grid technique to chance constrained optimal power flow. Generation, Transmission Distribution, IET 2013; 7(5):491-499.

[38] Tang J, Ni F, Ponci F, Monti A. Dimension-adaptive sparse grid interpolation for uncertainty quantification in modern power systems: Probabilistic power flow. Power Systems, IEEE Transactions on 2015; PP(99):1-13.

[39] Scientific Computing in Electrical Engineering SCEE 2010, Michielsen B, Poirier JR (eds.). Mathematics in Industry, 2012.

[40] Parrilo P, Lall S, Paganini F, Verghese GC, Lesieutre B, Marsden J. Model reduction for analysis of cascading failures in power systems. American Control Conference, 1999. Proceedings of the 1999, vol. 6, 1999; 4208-4212 vol.6.

[41] Chinesta F, Ladeveze P, Cueto E. A Short Review on Model Order Reduction Based on Proper Generalized Decomposition. Archives of Computational Methods in Engineering Oct 2011; 18(4):395-404.

[42] Chinesta F, Ammar A, Cueto E. Recent advances and new challenges in the use of the proper generalized decomposition for solving multidimensional models. Archives of Computational methods in Engineering 2010; 17(4):327-350.

[43] Chinesta F, Leygue A, Bordeu F, Aguado JV, Cueto E, Gonzalez D, Alfaro I, Ammar A, Huerta A. Pgd-based computational vademecum for efficient design, optimization and control. Archives of Computational Methods in Engineering Jan 2013; 20(1):31-59.

[44] Chinesta F, Leygue A, Bordeu F, Aguado J, Cueto E, Gonzalez D, Alfaro I, Ammar A, Huerta A. PGD-based computational vademecum for efficient design, optimization and control. Archives of Computational Methods in Engineering 2013; 20(1). 
[45] Mozolevski I, Prudhomme S. Goal-oriented error estimation based on equilibrated-flux reconstruction for finite element approximations of elliptic problems. Computer Methods in Applied Mechanics and Engineering 2015; 288:127 - 145.

[46] Florentin E, Díez P. Adaptive reduced basis strategy based on goal oriented error assessment for stochastic problems. Computer Methods in Applied Mechanics and Engineering 2012; 225-228:116 - 127.

[47] Martinez JA, Guerra G. Optimum placement of distributed generation in three-phase distribution systems with time varying load using a Monte Carlo approach. Power and Energy Society General Meeting, 2012 IEEE, IEEE, 2012; 1-7.

[48] Lambert T, Gilman P, Lilienthal P. Micropower System Modeling with HOMER. John Wiley, 2006. 